\title{
On Skolemization in constructive theories
}

\author{
Matthias Baaz* \\ Inst. for Discrete Math. and Geometry E104 \\ Technical University Vienna \\ Wiedner Hauptstrasse 8-10 \\ 1040 Vienna, Austria \\ (baaz@logic.at)
}

\author{
Rosalie Iemhoff \\ Department of Philosophy \\ University Utrecht \\ Heidelberglaan 6-8 \\ 3584 CS Utrecht, The Netherlands \\ (Rosalie.Iemhoff@phil.uu.nl)
}

February 6, 2008

\begin{abstract}
In this paper a method for the replacement, in formulas, of strong quantifiers by functions is introduced that can be considered as an alternative to Skolemization in the setting of constructive theories. A constructive extension of intuitionistic predicate logic that captures the notions of preorder and existence is introduced and the method, orderization, is shown to be sound and complete with respect to this logic. This implies an analogue of Herbrand's theorem for intuitionistic logic. The orderization method is applied to the constructive theories of equality and groups.
\end{abstract}

\section{Introduction}

Although seldomly mentioned, Skolemization is a method often used in mathematics. In computer science, where its presence is more conspicuous, it is at the basis of many an application. In mathematics Skolemization is mostly applied in the replacement of an axiom of the form $\forall x \exists y A(x, y)$ by an axiom of the form $\forall x A(x, f x)$ for a new function $f$ that is added to the language. That this operation does not change the theory follows from the fact that

$$
\forall x \exists y A(x, y) \vdash B \Leftrightarrow \forall x A(x, f x) \vdash B .
$$

In computer science, Skolemization often appears in combination with Herbrand's theorem, which states that for a formula $\exists y_{1} \ldots \exists y_{n} A\left(y_{1}, \ldots, y_{n}\right)$, where $A$ contains no quantifiers, there are terms $t_{11} \ldots t_{m n}$ such that

$$
\vdash \exists y_{1} \ldots \exists y_{n} A\left(y_{1}, \ldots, y_{n}\right) \Leftrightarrow \vdash \bigvee_{i=1}^{m} A\left(t_{i 1}, \ldots, t_{i n}\right)
$$
\footnotetext{
edged.

* Support by the Austrian Science Fund FWF under project P17503 is gratefully acknowl-
} 
Namely, by first applying Skolemization to a formula $B$ that is of the form $\exists y_{1} \forall x_{1} \ldots \exists y_{n} \forall x_{n} A\left(y_{1}, \ldots, y_{n}\right)$, where $A$ is quantifier free, we obtain a correspondce between predicate and propositional logic that, although easy to prove, is of fundamental importance:

$$
\vdash B \Leftrightarrow \vdash \bigvee_{i=1}^{m} A\left(t_{i 1}, f_{1}\left(t_{i 1}\right), t_{i 2}, f_{2}\left(t_{i 1}, t_{i 2}\right), \ldots, t_{i n}, f_{n}\left(t_{i 1}, \ldots, t_{i n}\right)\right)
$$

It has been known for a long time that there is no such correspondence in intuitionistic logic, because although sound, Skolemization fails to be complete for this logic; we do have

$$
\vdash_{\mathrm{IQC}} A \Rightarrow \vdash_{\mathrm{IQC}} A^{S} \text {, }
$$

in fact, we even have $\vdash_{\mathrm{IQC}}\left(A \rightarrow A^{S}\right)$, but in general the converse does not hold,

$$
\vdash_{\mathrm{IQC}} A^{S} \nRightarrow \vdash_{\mathrm{IQC}} A \text {. }
$$

Here $A^{S}$ is the Skolemization of $A$. Examples of this phenomenon are

$$
\nvdash_{\mathrm{IQC}} \neg \neg \exists x A x \rightarrow \exists x \neg \neg A x \quad \vdash_{\mathrm{IQC}} \neg \neg A c \rightarrow \exists x \neg \neg A x,
$$

and, in the context of axioms,

$$
\begin{aligned}
& \forall x \exists y A(x, y) \nvdash_{\mathrm{IQC}} \forall x \forall y \exists u \exists v(A(x, u) \wedge A(y, v) \wedge(x=y \rightarrow u=v)), \\
& \forall x A(x, f x) \vdash_{\mathrm{IQC}} \forall x \forall y \exists u \exists v(A(x, u) \wedge A(y, v) \wedge(x=y \rightarrow u=v)) .
\end{aligned}
$$

The last example indicates that in a constructive setting replacing an axiom $\forall x \exists y A(x, y)$ by $\forall x A(x, f x)$ does not create a conservative extension of the original theory, as is the case for classical theories. Observe that in the two examples above Skolemization is not restricted to prenex formulas, but is applied to infix formulas as well. Of course, in the context of intuitionistic logic we have to extend the Skolemization method to such formulas because in this logic not every formula has a prenex normal form. Therefore, in this setting Skolemization means the replacement of strong quantifiers, i.e. positive universal and negative existential quantifiers, by functions, as can be seen in the examples above.

The class of formulas for which Skolemization is complete is well-understood, mostly due to beautiful work by Grigori Mints who characterized a large part of this class and proved many results on the subject, including Herbrand-like theorems for intuitionistic logic $[10,11,12,14,15]$. Another line of research focusses on automated theorem proving in intuitionistic logic. Here one analyzes the cases in which a proof of $A^{S}$ can be transformed into a proof of $A$, as in the papers by Fitting [8], Kreitz et al. [9], Mints [13], Shankar [17] and Wallen [19]. This work has many applications and contains deep insights, especially on the proof theory of intuitionistic logic. 


\subsection{Orderization}

In this paper, like in the work by Mints, we approach the problem from a less application orientated and more foundational point of view. We start by analyzing the reasons for the incompleteness of Skolemization in a constructive setting. As it turns out, the fact that Skolemization fails to be complete for intuitionistic logic is related to the expressive power of the logic. We show that by extending intuitionistic logic by a preorder and an existence predicate we can define a translation, called orderization and denoted by ()$^{\circ}$, which can be viewed as a sound and complete alternative to Skolemization. That is, we define a logic IQCO with corresponding sequent calculus LJO, which are extensions of IQC and LJ, such that for all sequents $S$ in the original language

$$
\vdash_{\text {LJ }} S \Leftrightarrow \vdash_{i o} S \Leftrightarrow \vdash_{i o} S^{o} .
$$

Here $\vdash_{i o}$ stands for $\vdash_{\text {LJo. }}$ As in Skolemization, the method replaces strong quantifiers by functions in a canonical way, but it also uses the extra expressive power of the logic, i.e. the order relation and the existence predicate that are part of the language. Thus $S^{o}$ is a formula that contains no strong quantifiers but might contain these two additional predicates.

What is important is that IQCO is a constructive extension of IQC. The following observation might help the reader to appreciate this fact. The statement that a formula $A$ is true in intuitionistic logic, i.e. that it holds in all Kripke models, can be expressed in predicate logic, say by a formula $T(A)$. By Skolemizing this formula we obtain

$$
\vdash_{\mathrm{IQC}} A \Leftrightarrow \vdash_{\mathrm{CQC}} T(A) \Leftrightarrow \vdash_{\mathrm{CQC}}(T(A))^{S} .
$$

Besides being a trivial observation, this fact does not teach us anything about intuitionistic logic, which is our final aim. Also, the formula $T(A)$ will in general be huge even if $A$ is not, which makes the method less applicable and unelegant. In contrast, IQCO is a constructive theory and $A^{\circ}$ is fairly close to $A$.

There is not one unique answer to the problem of Skolemization in intuitionistic logic. One could take its failure at face value and not search for alternatives, or one could look for alternatives in other directions than the ones discussed above. Thus the answer given in this paper is just one possible answer, and in the future other approaches may emerge that prove fruitful as well.

\subsection{Theories}

After introducing the notion of orderization and proving the above mentioned results, the method will be applied to constructive theories. Here the situation changes slightly in that we have to orderize the theories as well. In classical logic Skolemization does not affect the theory at hand:

$$
T \vdash_{\mathrm{CQC}} A \Leftrightarrow T \vdash_{\mathrm{CQC}} A^{S} .
$$


In the case of orderization we obtain

$$
T \vdash_{\mathrm{LJ}} S \Leftrightarrow T \vdash_{i_{0}} S \Leftrightarrow T^{k} \vdash_{i o} S^{o},
$$

where $T^{k}$ is a translation of $T$. In fact, this translation is part of orderization in that $A^{o}$ is defined as $\left(A^{k}\right)^{S}$. In many cases, $T^{k}$ can be replaced by the theory itself, often with one extra axiom, as in the case of equality and groups.

The results on orderization easily imply analogues of Herbrand's theorem for theories $T$ for which $T^{k}$ is universal. It will be shown that for every sequent $S$ in the original language there exists a quantifier free $\wedge \vee$-expansion $S^{\prime}$ of $S^{o}$ such that

$$
T \vdash_{\mathrm{LJ}} S \Leftrightarrow T \vdash_{i o} S \Leftrightarrow T^{k} \vdash_{i o} S^{o} \Leftrightarrow T^{k} \vdash_{i o} S^{\prime} .
$$

As we will see, al theories discussed in this paper have decidable quantifier free fragments. Thus it is decidable whether the $\wedge \vee$-expansion of sequents of the form $S^{o}$ are derivable in the theory or not.

Finally, a remark on the proof methods in this paper. Skolemization and Herbrands's theorem have a strong proof theoretic flavour and are easy to prove for classical logic once the right proof system is considered. In fact, the proofs of many other results on Skolemization rely on proof theoretic methods as well. On the other hand, almost all proofs in this paper use models, even though IQCO has a well-behaved sequent calculus LJO. Proof theoretic proofs would be a worthwhile addition to these results, as they might lead the way to an algorithm that translates proofs of $S^{o}$ into proofs of $S$. However, that in the setting of Skolemization semantical arguments can lead to insights that are yet not clearly visible from the proof theoretic point of view is an interesting phenomenon in itself.

\section{Skolemization}

In this section we will analyze the failure of Skolemization in intuitionistic predicate logic IQC and from this observation derive the construction of an alternative Skolemization method called orderization. This section contains all the intuitions behind the results to come, which will be made precise and proved in the next sections.

We denote by $A^{S}$ the Skolemization of a formula $A$. That is, $A^{S}$ is the result of replacing every strong quantifier $Q x B(x, \bar{y})$ in $A$ by $B(f(\bar{y}), \bar{y})$, where $\bar{y}$ are the variables of the weak quantifiers in the scope of which $Q x B(x, \bar{y})$ occurs. We require that every Skolem function $f$ in $A^{S}$ is distinct from the others, is in $\mathcal{L}_{s}$, and does not occur in $A$.

Following Sam Buss in $[6,7]$, the Herbrandization $A^{H}$ of a formula is the result of replacing every weak quantifier $Q x B(x, \bar{y})$ in $A$ by $B(f(\bar{y}), \bar{y})$, where $\bar{y}$ are the variables of the strong quantifiers in the scope of which $Q x B(x, \bar{y})$ occurs. The same requirements as for $A^{S}$ apply. 
For a set of formulas $\Gamma, \Gamma^{S}$ denotes $\left\{A^{S} \mid A \in \Gamma\right\}$, and similarly for $H$. We denote by $S^{S}$ the Skolemization of the sequent $S=(\Gamma \Rightarrow \Delta)$, which is defined as $\Gamma^{H} \Rightarrow \Delta^{S}$, and analogous for $S^{H}$.

We have seen that Skolemization is sound but not complete for IQC, that is, $\vdash_{\mathrm{IQC}} A^{S}$ does not imply $\vdash_{\mathrm{IQC}} A$. From a semantical point of view this means that there are formulas $A$ that have a countermodel while their Skolemization $A^{S}$ has not, as for example the existential double negation shift and the universal principle of excluded middle:

$$
\begin{array}{rrr}
E D & \nvdash_{\mathrm{IQC}} \neg \neg \exists x P x \rightarrow \exists x \neg \neg P x & \vdash_{\mathrm{IQC}} \neg \neg P c \rightarrow \exists x \neg \neg P x \\
U P & \nvdash_{\mathrm{IQC}} \neg \neg \forall x(P x \vee \neg P x) & \vdash_{\mathrm{IQC}} \neg \neg(P c \vee \neg P c) .
\end{array}
$$

Examples of countermodels to these formulas are given in the following picture.

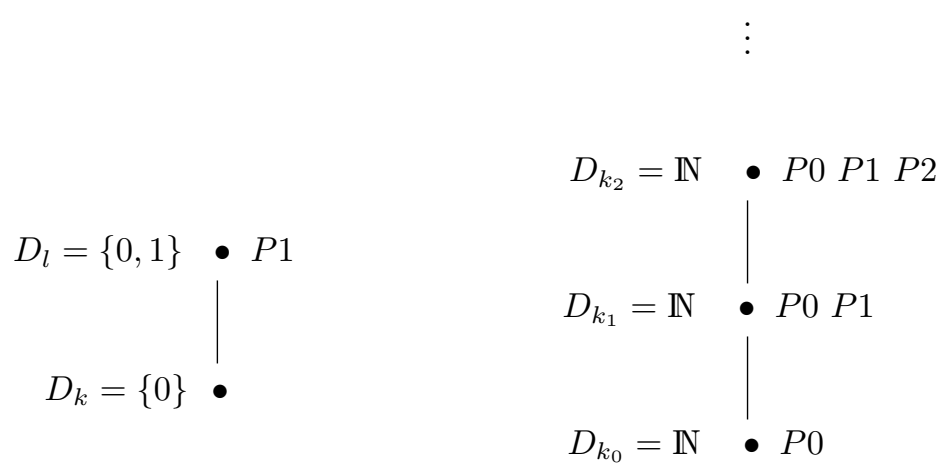

Countermodel $M$ to $E D$

Countermodel $N$ to $U P$

We know that it is impossible to make these models into countermodels of $E D^{S}$ and $U P^{S}$ because these formulas are derivable. The following could be considered as the "reasons" for this failure, reasons that are different for $M$ and $N$. In $M$, the fact that 1 is not an element of the domain in the root blocks the possibility that in this node we could interpret the Skolem constant $c$ as 1 and force $\neg \neg P c$. In $N$, no number $n$ in the domain $\mathbb{N}$ can serve as the interpretation of the $c$ in $\neg \neg(P c \vee \neg P c)$, as eventually $P n \vee \neg P n$ will be forced, and thus $\neg \neg(P c \vee \neg P c)$ would be forced in the root.

\subsection{The existence predicate}

How could we extend the expressive power of intuitionistic logic in such a way that we could transform the countermodels $M$ and $N$ of $E D$ and $U P$ into countermodels of $E D^{S}$ and $U P^{S}$ ? That is, is there a constructive extension of IQC such that using the increased expressive power, an analogue of Skolemization can 
be defined. An analogue for which completeness can be proved by transforming countermodels of a formula into countermodels of its Skolemized counterpart, i.e. of the formula that is the translation of the original formula under this alternative of Skolemization. What we are after can best be explained by a solution to the problem for existential quantifiers that the authors proposed in [4]. In that paper an extension IQCE of IQC first introduced by Dana Scott [16] was used. This logic is intuitionistic logic extended by a so-called existence predicate $E$, a unary predicate that denotes that a term exists, the idea being that the existence predicate has to be included in quantified statements. For example, to conclude $\exists x A x$ both $A t$ and $E t$ should be established for some term $t$. A sequent calculus LJE $[4,3,1,2]$ for this logic consists of the rules and connectives of $\mathrm{LJ}$ and the following quantifier rules.

$$
\begin{array}{ll}
\mathrm{L} \forall \frac{\Gamma, A t \Rightarrow C \quad \Gamma, \forall x A x \Rightarrow E t}{\Gamma, \forall x A x \Rightarrow C} & \mathrm{R} \forall \frac{\Gamma, E y \Rightarrow A y}{\Gamma \Rightarrow \forall x A[x / y]} * \\
\mathrm{~L} \exists \frac{\Gamma, A y, E y \Rightarrow C}{\Gamma, \exists x A[x / y] \Rightarrow C} * & \mathrm{R} \exists \frac{\Gamma \Rightarrow A t \quad \Gamma \Rightarrow E t}{\Gamma \Rightarrow \exists x A x}
\end{array}
$$

The semantics of IQC has to be adapted accordingly. For example, $k \Vdash \exists x A x$ holds if and only if $k \Vdash E d \wedge A d$ for some element $d \in D_{k}$. LJE is sound and complete with respect to this semantics. It is not difficult to see that in this case one can restrict the semantics to models with constant domains, because the existence predicate cuts out the existing elements at every node seperately. Because of this, the problem with model $M$ above disappears: every element has a name in the root but might come into existence at a later point only. By interpreting $c$ as 1, the following pictures presents a countermodel to $E D^{s}$.

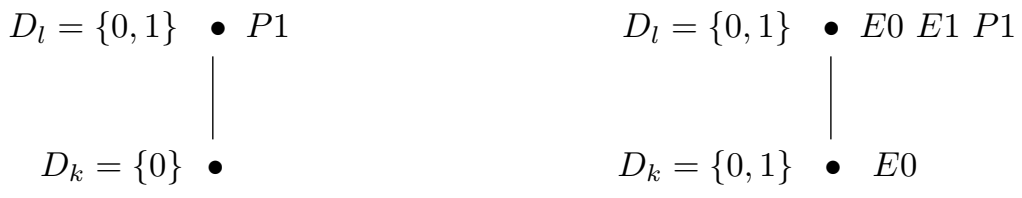

Countermodel $M$ to $E D \quad$ Countermodel to $E D^{s}$ in IQCE

The right side is a countermodel to $E D^{s}$ because the root forces $\neg \neg P 1$ but not $\exists x \neg \neg P x$ because $E 1$ does not hold in the root.

The precise result on this logic with existence is as follows. In this context the ESkolemization $A^{s}$ of $A$ is the result of replacing every strong quantifier $Q x B(x)$ in $A_{i}$ by

$$
E f(\bar{y}) \rightarrow B(f(\bar{y})) \text { if } Q=\forall
$$

and by

$$
E f(\bar{y}) \wedge B(f(\bar{y})) \text { if } Q=\exists .
$$


Then it can be shown that for a set of axioms $\mathcal{A}$ of the form $E t$, ESkolemization can indeed be viewed as an alternative to Skolemization (we skip some details):

Theorem 1 [4] For each closed sequent $S$ in which all strong quantifiers are existential:

$$
\vdash_{\text {LJ }} S \Leftrightarrow \mathcal{A} \vdash_{\text {LJE }} S \Leftrightarrow \mathcal{A} \vdash_{\text {LJE }} S^{s} .
$$

In fact, stronger results than these have been proved in [4], but this theorem suffices to explain the intuition on countermodels discussed above. Indeed, the ESkolemization $E D^{s}$ of $E D$ is the formula

$$
\neg \neg(E c \wedge P c) \rightarrow \exists x \neg \neg P x .
$$

which, like $E D$ itself, is not derivable in LJE. Since IQCE is a natural and constructive extension of IQC, ESkolemization could be considered as an analogue or alternative to Skolemization for constructive settings. But, alas! the method only applies to existential quantifiers. In the next section we show how the universal quantifiers can be dealt with.

\subsection{Orderization}

It is not difficult to see that the above technique of transforming countermodels of formulas to countermodels of their Skolemization or ESkolemization does not appply to universal quantifiers. Indeed, the ESkolemization $\neg \neg(E c \rightarrow P c \vee \neg P c)$ of $U P$ is still derivable. Here the infinity of the model plays a role, as any finite model forces UP. The idea is that this infinity should be expressible in the logic in order to be able to Skolemize universal quantifiers. Of course, there are many ways to do this, for example by using infinite disjunctions and conjunctions, or by using second-order logic. Recall however that our aim is to stay as close to intuitionistic predicate logic as possible and remain constructive as well. As it turns out, surprisingly little is needed to make the intuition about representing infinity work. Besides the existence predicate we extend the language by a preorder $\preccurlyeq$ and a binary function $\langle$,$\rangle . Intuitively, expressions A\langle k, d\rangle$ denote that $A d$ is forced at node $k$. The axioms we add to IQC state that $\preccurlyeq$ is a preorder and that upwards persistency holds for expressions $A\langle k, d\rangle$. In fact, we do less, but for the sake of clarity we omit some details here. The resulting system is called IQCO, the $O$ for order. Like IQCE, IQCO has a simple sequent calculus LJO which is sound and complete for Kripke models of IQCO. Moreover it clearly is a constructive logic, and possesses many of the properties one would wish sych a system to have, like the disjunction property and the existence property.

Using this expressive power of IQCO an analogue of Skolemization is introduced, called orderization. The orderization $A^{o}$ of a formula $A$ is obtained in two steps. First, subformulas $(A \rightarrow B)$ are replaced by $\forall l \succcurlyeq k(A\langle l, x\rangle \rightarrow B\langle l, x\rangle)$, and subformulas $\forall x A x$ by $\forall l \succcurlyeq k \forall x(E\langle l, x\rangle \rightarrow A\langle l, x\rangle)$, and the result is called $A^{k}$ (again, we skip a lot of details here to make the main argment clear). Second, 
classical Skolemization is applied and the result is called $A^{o}$. Thus $A^{o}=\left(A^{k}\right)^{S}$. Note the similarity between the $(\cdot)^{k}$ translation and the forcing conditions for implication and universal quantification in Krikpke models. It is as Lev Beklemished once pointed out: in intuitionistic logic implication should be Skolemized too.

For the orderization translation the results mentioned in the introduction will be proved in the subsequent sections. Hopefully they convince the reader that orderization can indeed be seen as, maybe not the, but certainly a reasonable analogue of Skolemization in a constructive setting.

\section{The logics}

As explained in the introduction, we will mainly work with a constructive extension of intuitionistic logic, IQCO, that contains axioms for certain specific predicates and functions in the langauge. To distinguish the Skolem functions from our original language we use three languages $\mathcal{L}, \mathcal{L}_{o}$ and $\mathcal{L}_{s}$ that are assumed to be disjunct. $\mathcal{L}$ can be any language for first-order logic. $\mathcal{L}_{s}$, the Skolem language, constains infinitely many variables $k, l, \ldots$, and for every arity infinitely many functions. Constants are treated as nullary functions. $\mathcal{L}_{o}$ consists of the constants $\epsilon, \iota$, unary predicates $K$ and $E$, a binary predicate $\preccurlyeq$, and a binary function $\langle\cdot, \cdot\rangle$. Thus in this case we do not only have Skolem functions but also three distinguished predicates, two distinguished constants and a distinguished function. Intuitively, these symbols enable the description of a Kripke model, or at least part of it: $K$ is the set of nodes, $\preccurlyeq$ is the preorder, $\iota$ is the root, $\epsilon$ an element in the domain of the root, $E$ an existence predicate that describes the domains of the nodes, and the function $\langle$,$\rangle that expresses at which node a$ given formula is evaluated. As mentioned above, $A\langle k, t\rangle$ describes that " $A t$ is forced at node $k "$.

Some words on notation. For $n$-ary formulas $A(\bar{x})$ and $\bar{a}=a_{1}, \ldots, a_{n}$ we use the following abbreviation:

$$
A\langle k, \bar{a}\rangle \equiv_{\text {def }} A\left(\left\langle k, a_{1}\right\rangle, \ldots,\left\langle k, a_{n}\right\rangle\right) .
$$

To improve transparancy we sometimes suppress atomic formulas of the form $K k$ in a formula: in most situations $\forall l \succcurlyeq k A(l)$ is short for $\forall l(K l \wedge l \succcurlyeq k \rightarrow A(l))$. We will remind the reader of this convention at several points in the paper. We write $A \in \mathcal{L}$ if $A$ is a formula in the language $\mathcal{L}$, and likewise for the other languages.

\subsection{Gentzen calculi}

In this section we introduce the Gentzen calculi on which all theories that are discussed in this paper are based. First we recall the Gentzen calculus LJ for intuitionistic predicate logic, and then we extend it to the calculus LJO that includes the extra axioms that we discussed in the introduction. In the next 
sequent we discuss the Kripke models for this logic. Sequents are expressions of the form $\Gamma \Rightarrow \Delta$, where $\Gamma$ and $\Delta$ are multisets. $\Gamma$ and $\Delta$ will always range over multisets of formulas.

$$
I(\Gamma \Rightarrow \Delta) \equiv_{\text {def }} \bigwedge \Gamma \rightarrow \bigvee \Delta
$$

\section{The system LJ}

$$
\begin{aligned}
& \text { Ax } P \Rightarrow P(P \text { a predicate }) \quad L \perp \perp \Rightarrow \\
& \mathrm{RW} \frac{\Gamma \Rightarrow}{\Gamma \Rightarrow A} \\
& \mathrm{LW} \frac{\Gamma \Rightarrow C}{\Gamma, A \Rightarrow C} \\
& \mathrm{~L} \wedge \frac{\Gamma, A, B \Rightarrow C}{\Gamma, A \wedge B \Rightarrow C} \\
& \mathrm{R} \wedge \frac{\Gamma \Rightarrow A \quad \Gamma \Rightarrow B}{\Gamma \Rightarrow A \wedge B} \\
& \mathrm{~L} \vee \frac{\Gamma, A \Rightarrow C \quad \Gamma, B \Rightarrow C}{\Gamma, A \vee B \Rightarrow C} \\
& \mathrm{R} \vee \frac{\Gamma \Rightarrow A_{i}}{\Gamma \Rightarrow A_{0} \vee A_{1}} i=0,1 \\
& \mathrm{~L} \rightarrow \frac{\Gamma \Rightarrow A, C \quad \Gamma, B \Rightarrow C}{\Gamma, A \rightarrow B \Rightarrow C} \\
& \mathrm{R} \rightarrow \frac{\Gamma, A \Rightarrow B}{\Gamma \Rightarrow A \rightarrow B} \\
& \mathrm{~L} \forall \frac{\Gamma, A t \Rightarrow C}{\Gamma, \forall x A x \Rightarrow C} \\
& \mathrm{R} \forall \frac{\Gamma \Rightarrow A y}{\Gamma \Rightarrow \forall x A[x / y]} * \\
& \mathrm{~L} \exists \frac{\Gamma, A y \Rightarrow C}{\Gamma, \exists x A[x / y] \Rightarrow C} * \\
& \mathrm{R} \exists \frac{\Gamma \Rightarrow A t}{\Gamma \Rightarrow \exists x A x} \\
& \text { Cut } \frac{\Gamma \Rightarrow A \quad \Gamma, A \Rightarrow C}{\Gamma \Rightarrow C} \\
& \text { LC } \frac{\Gamma, A, A \Rightarrow C}{\Gamma, A \Rightarrow C}
\end{aligned}
$$

Here $(*)$ denotes the condition that $y$ does not occur free in $\Gamma$ and $C$. LK is the classical version of LJ, that is, LJ in which the formula $C$ is replaced by $\Delta$, and $\Delta$ 's are added to the succedents of the right rules, and to which contraction at the right is added:

$$
\mathrm{RC} \frac{\Gamma \Rightarrow A, A, \Delta}{\Gamma \Rightarrow A, \Delta}
$$

We write LJ $\vdash S$ if the sequent $S$ is derivable in LJ. For a set of sequents $\mathcal{S}$, we say that $S$ is derivable from $\mathcal{S}$ in $\mathrm{LJ}$, and write $\mathcal{S} \vdash_{\mathrm{LJ}} S$, if $S$ is derivable in LJ extended by the axioms $\mathcal{S}$. Similarly for LK. 
The system LJ is extended by axioms defining a preorder that we will use in the definition of orderization to come. One should keep in mind that the special Skolem symbols are used to describe Kripke models, as explained in the introduction.

\subsection{Axiom for preorder}

The following axioms describe that $\preccurlyeq$ is a preorder on $K$ with root $\iota$.

$$
\begin{aligned}
A x_{p o} \quad \equiv_{\text {def }} & K k \Rightarrow k \preccurlyeq k \\
& K k, K k^{\prime}, K k^{\prime \prime}, k \preccurlyeq k^{\prime}, k^{\prime} \preccurlyeq k^{\prime \prime} \Rightarrow k \preccurlyeq k^{\prime \prime} \\
& \Rightarrow K \iota \\
& K k \Rightarrow \iota \preccurlyeq k .
\end{aligned}
$$

\subsection{Axiom for existence}

The following axioms are used to describe the domain of a node via the existence predicate and the binary function $\langle\rangle:, E\langle k, d\rangle$ corresponds to $d \in D_{k}$. The constant $\epsilon$ is interpreted as an element of the domain at the root, which therefore is not empty.

$$
\begin{aligned}
A x_{e x} \quad \equiv_{\text {def }} & \Rightarrow E\langle\iota, \epsilon\rangle \\
& K k, E\left\langle k, x_{1}\right\rangle, \ldots, E\left\langle k, x_{n}\right\rangle \Rightarrow E\left\langle k, f\left(x_{1}, \ldots, x_{n}\right)\right\rangle \quad(f \in \mathcal{L}) .
\end{aligned}
$$

Note that the last axiom implies $\Rightarrow E\langle\iota, t\rangle$ and $K k \Rightarrow E\langle k, t\rangle$ for every closed term $t$ in $\mathcal{L} \cup\{\epsilon\}$. Intuitively the axiom means that if $\bar{d}$ belongs to $D_{k}$, then so does $f(\bar{d})$. Observe that these axioms do not imply that $E\langle k, d\rangle \Rightarrow E\langle l, d\rangle$ for $k \preccurlyeq l$, which should hold if an expression $E\langle k, d\rangle$ is meant to decribe the domain $D_{k}$. As we will see, the next axiom implies just that.

\subsection{Axiom for upwards persistency}

As mentioned above, $A\langle k, d\rangle$ is meant to describe that $A d$ is forced at node $k$. The following axiom describes the upwards persistency in Kripke models, only for atomic formulas, which, as we will see later, suffices.

$$
A x_{u p} \equiv_{\text {def }} P\langle k, \bar{x}\rangle, E\langle k, \bar{x}\rangle, K k, K l, k \preccurlyeq l \Rightarrow P\langle l, \bar{x}\rangle \quad(P \in \mathcal{L} \text { or } P=E) .
$$

Observe that this implies $k \preccurlyeq l, E\langle k, d\rangle \Rightarrow E\langle l, d\rangle$, the property that was commented on above.

Define

$$
A x_{o} \equiv_{d e f} A x_{p o} \cup A x_{e x} \cup A x_{u p} .
$$

The logics IQCO and CQCO are IQC and CQC extended by the $I(S)$ of all $S \in A x_{o}$. As usual, the axioms are presented as open formulas (or sequents), but when applied in a proof their universally quantified version is used.

Observe that strictly speaking IQCO and CQCO are not logics but theories, as they are not closed under substitution. 


\subsection{Calculi for preorders}

Let LJO be the calculus LJ to which the axioms $A x_{o}$ have been added. We write $\vdash_{i o}$ for $\vdash_{\text {LJO }}$. Let LKO be the calculus LK extended by the axioms $A x_{o}$. We write $\vdash_{c o}$ for $\vdash_{\text {LKO }}$. In the section on completeness we will prove that for all sequents $S \in \mathcal{L}: \vdash_{\mathrm{LJ}} S \Leftrightarrow \vdash_{i o} S$ and $\vdash_{\mathrm{LK}} S \Leftrightarrow \vdash_{c o} S$, which follows naturally from the fact that $\mathcal{L}$ does not contain the Skolem predicates $\preccurlyeq, K$, and $E$, and the Skolem functions $\iota, \epsilon$, and $\langle$,$\rangle .$

Lemma 1 (Atomic Cut Lemma)

For every proof in LJO or LKO there is a proof in the same system of the same endsequent that only contains cuts which cutformulas are atomic.

In Section 9 it will be shown that the quantifier free fragments of these calculi are decidable, and that the calculi have the disjunction property

$$
\mathcal{T} \vdash A \vee B \Rightarrow \mathcal{T} \vdash A \text { or } \mathcal{T} \vdash B,
$$

and the term existence property

$$
\mathcal{T} \vdash \exists x A(x) \Rightarrow \exists t(t \text { a term }) \mathcal{T} \vdash A(t) .
$$

\section{The semantics}

The semantics of the logics IQCO and CQCO are based on Kripke models. Here we briefly recall what these are and fix notation. To avoid variable clashes in what follows, the language of Kripke models is always assumed to be disjunct from $\mathcal{L}$ and $\mathcal{L}_{s}$ unless the models are explicitly constructed in terms of these languages. The models are defined for the full language $\mathcal{L} \cup \mathcal{L}_{o} \cup \mathcal{L}_{s}$. If $\mathcal{L}$ contains equality it is treated as any other binary predicate for the moment. It will play a special role, but only later so.

\subsection{Kripke models}

A classical model is a pair $M=(D, I)$ such that $D$ is a set and $I$ is a map such that

$I(P)$ is an $n$-ary predicate on $D$ for every $n$-ary predicate $P$,

$I(f)$ is an $n$-ary function on $D$ for every $n$-ary function $f$ (constants are treated as 0-ary functions).

$I(t)$ denotes the interpretation of a term $t$ under $I$, which is defined as usual. $I\left(t_{1}, \ldots, t_{n}\right)$ is short for $I\left(t_{1}\right), \ldots, I_{k}\left(t_{n}\right)$. For sentences $A$, let $(D, I) \models A$ denote that $A$ holds in the model $(D, I)$, which is defined as usual for classical models. A frame is a pair $(W, \preccurlyeq)$ where $W$ is a nonempty subset of $\mathcal{K}$ and $\preccurlyeq$ is a partial order on $W$ with a root. A Kripke model on a frame $F=(W, \preccurlyeq)$ is a quadruple 
$K=(W, \preccurlyeq, D, I)$, where $D$ is a collection $\left\{D_{k} \mid k \in W\right\}$ of nonempty sets called domains that satisfy $D_{k} \subseteq D_{l}$ if $k \preccurlyeq l$. I is a collection $\left\{I_{k} \mid k \in W\right\}$ such that the $\left(D_{k}, I_{k}\right)$ are classical models that satisfy the persistency requirement: for all $k \in W$, for all predicates $P(\bar{x})$ and for all $\bar{c} \in D_{k}$, and all terms $t$,

$$
\begin{gathered}
k \preccurlyeq l \Rightarrow\left(\left(D_{k}, I_{k}\right) \models P(\bar{c}) \Rightarrow\left(D_{l}, I_{l}\right) \models P(\bar{c})\right), \\
k \preccurlyeq l \Rightarrow I_{k}(t)=I_{l}(t) .
\end{gathered}
$$

In particular, $I_{k}(t)=I_{l}(t)$ for all $k$ and $l$ since the models are rooted.

Observe that the symbols $K$ and $\preccurlyeq$ are used both as the Skolem predicates in $\mathcal{L}_{s}$ and as the name of a model and its assecibility relation. We find this ambiguity convenient as it suggests a connection that indeed exists in many theorems to come. At places where it could cause confusion we will be explicit about which interpretation of the symbols is meant.

Given a Kripke model $K=(W, \preccurlyeq, D, I)$ the forcing relation is defined as usual: for predicates $P(\bar{x})$, terms $\bar{t}(\bar{x})$ and $\bar{d} \in D_{k}$, we put

$$
K, k \Vdash P(t(\bar{d})) \equiv_{\text {def }}\left(D_{k}, I_{k}\right) \models P(t(\bar{d})),
$$

and extend $K, k \Vdash A$ to all sentences in the usual way:

$$
\begin{aligned}
& k \Vdash \perp \quad k \Vdash \top \\
& k \Vdash A \wedge B \text { iff } k \Vdash A \text { and } k \Vdash B \\
& k \Vdash A \vee B \text { iff } k \Vdash A \text { or } k \Vdash B \\
& k \Vdash A \rightarrow B \text { iff } \forall l \succcurlyeq k: l \Vdash A \Rightarrow l \Vdash B \\
& k \Vdash \exists x A(x) \text { iff } \exists d \in D_{k} k \Vdash A(d) \\
& k \Vdash \forall x A(x) \text { iff } \forall l \succcurlyeq k \forall d \in D_{l} l \Vdash A(d) .
\end{aligned}
$$

When $K$ is clear from the context we write $k \Vdash A$ instead of $K, k \Vdash A$. We say that $A$ is forced in $K, K \Vdash A$, if for all nodes $k, K, k \Vdash A$. For a formula $A(\bar{x})$, $K \Vdash A(\bar{x})$ if $K \Vdash A[\bar{a} / \bar{x}]$ for all $\bar{a} \in D$. We use the same notation for sequents, where it is understood that $k \Vdash S$ if and only if $k \Vdash I(S)$. Observe that $=$, if present in $\mathcal{L}$, is interpreted in the models as an arbitrary binary predicate, because interpreting it as real equality would make it decidable.

\subsection{Ipomodels and cpomodels}

An ipomodel (intuitionistic preorder model) is a Kripke model that satisfies the axioms of LJO, i.e. $A x_{o}$. A cpomodel (classical preorder model) is a classical model that satisfies the axioms of LKO, i.e. $A x_{o}$. We write $\Vdash_{i o} A$ when $A$ is forced in all ipomodels. We write $\mathcal{S} \Vdash_{i o} S$ if $I(S)$ if forced in all ipomodels that force all formulas $I\left(S^{\prime}\right)$ for $S^{\prime} \in \mathcal{S}$. For cpomodels, $M \models A$ and the like are defined as usual for classical models. We write $\models_{c o} S$ if $S$ holds in all cpomodels. 


\subsection{Soundness and completeness of the proof systems}

Theorem 2 For sets of closed sequents $\mathcal{S}$ and closed sequents $S$ :

$$
\mathcal{S} \vdash_{i o} S \text { iff } \mathcal{S} \vdash_{i o} S \quad \mathcal{S} \vdash_{c o} S \text { iff } \mathcal{S} \models_{c o} S .
$$

Proof The directions from left to right are clear. For the other direction, recall that $\vdash_{i o}$ is equal to $A x_{o} \vdash \mathrm{LJ}$. The completeness of LJ now implies the result. Similarly for LKO.

Corollary 1 For sets of closed sequents $\mathcal{S}$ and closed $S$, both in $\mathcal{L}$ :

$$
\mathcal{S} \vdash_{\mathrm{LJ}} S \Leftrightarrow \vdash_{i_{0}} S \quad \mathcal{S} \vdash_{\mathrm{LK}} S \Leftrightarrow \vdash_{c o} S .
$$

Proof The directions from left to right are clear. For the other direction of the first equivalence, suppose $M$ is a Kripke model with root $r$ that refutes $S$. From $M$ we construct an ipomodel $N$ that refutes $S$ as follows. $N$ is equal to $M$ except for the interpretation of $E, K, \preccurlyeq, \iota, \epsilon$ and $\langle$,$\rangle , which are defined as$ follows. Fix an element $d \in D_{r}$. In every node, $\iota$ and $\epsilon$ are interpreted as $d,\langle$, is interpreted as the constant function $d, K$ and $E$ only hold on $d$ and $\preccurlyeq$ only on $(d, d)$. It is not difficult to see that $N$ is an ipomodel that forces the same formulas in $\mathcal{L}$ as $M$. Similar reasoning applies to the classical case.

Note that in the corollary the assumption that $\mathcal{S}$ and $S$ are in $\mathcal{L}$ could be replaced by the assumption that they do not contain elements in $\mathcal{L}_{o}$.

\section{Translations}

We define a translations $(\cdot)^{k}$ from formulas to formulas which will translate formulas provable in LJ to formulas provable in LJO. Intuitively, $A^{k}$ describes that $k \Vdash A$. The challenge is to achieve this with the limited means that we have, that is, with the few extra Skolem symbols that we have available in $\mathcal{L}_{o}$. For a sequence of terms $\bar{t}=t_{1}, \ldots, t_{n}$, we write $\left(\bar{t}^{k}\right.$ or $\bar{t}^{k}$ for the sequence $t_{1}^{k}, \ldots, t_{n}^{k}$. Recall that we use the abbreviation $\forall l \succcurlyeq k A$ for $\forall l(K l \wedge l \succcurlyeq k \rightarrow A)$, and that $A\langle k, \bar{x}\rangle$ is short for $A\left(\left\langle k, x_{1}\right\rangle, \ldots,\left\langle k, x_{n}\right\rangle\right)$.

The translation is inductively defined as follows.

$$
\begin{aligned}
& \perp^{k}=\perp \text { and } \top^{k}=\top, \\
& t^{k}=\langle k, t\rangle \text { for terms } t, \\
& (P(\bar{t}))^{k}=P\left(\bar{t}^{k}\right) \text { for predicates } P, \\
& (\cdot)^{k} \text { commutes with } \wedge \text { and } \vee, \\
& (B \rightarrow C)^{k}=\forall l \succcurlyeq k\left(B^{l} \rightarrow C^{l}\right), \\
& (\exists x A(x))^{k}=\exists x\left(E\langle k, x\rangle \wedge A(x)^{k}\right),
\end{aligned}
$$




$$
(\forall x A(x))^{k}=\forall l \succcurlyeq k \forall x\left(E\langle l, x\rangle \rightarrow A(x)^{l}\right) .
$$

To avoid variable clashes we require that all variables added in the translation are distinct and differ from all variables in the original formula. Strictly speaking, the definition above is the definition of a translation on formulas up to equivalence. We will often write $A^{k} t$ for $(A(t))^{k}$.

Clearly,

$$
(P(t))^{k}=P\langle k, t\rangle \quad(P(s, f t))^{k}=P(\langle k, s\rangle,\langle k, f t\rangle),
$$

and similarly for predicates of arbitrary arity. Note that if $A$ is in $\mathcal{L}$ and $K l$ occurs in $A^{k}$, then for $l \neq k, l$ is not free in $A^{k}$. Also, for any connective $\circ$, no subformula in $A^{k}$ is of the form $\left(B^{l} \circ C^{m}\right)$ unless $l=m$. For sequents we define

$$
(\Gamma \Rightarrow \Delta)^{k} \equiv_{\text {def }} K k, \Gamma^{k} \Rightarrow \Delta^{k} .
$$

Example 1 For predicates $P$ and $Q$ we have

1. $(P(t) \rightarrow Q(x, y))^{k}=\forall l \succcurlyeq k(P\langle l, t\rangle \rightarrow Q(\langle l, x\rangle,\langle l, y\rangle))$,

2. $(\forall x P(x, \bar{y}))^{k}=\forall l \succcurlyeq k \forall x(E\langle l, x\rangle \rightarrow P\langle l, x, \bar{y}\rangle)$.

3. $(\neg A)^{k}=\forall l \succcurlyeq k \neg A^{l}$,

4. $(\neg \neg A)^{k}=\forall l \succcurlyeq k \neg \forall m \succcurlyeq l \neg A^{m} \equiv \forall l \succcurlyeq k \neg \neg \exists m \succcurlyeq l A^{m}$.

Observe that $\neg\left(A^{k}\right)$ is not of the form $B^{l}$, although in some cases it might be equivalent to it.

By formula induction the following lemma can be proved easily.

Lemma 2 For a formula $A(\bar{s})$ in which $K$ and $\preccurlyeq$ do not occur, and in in which $\bar{s}$ are the only terms,

$$
\vdash_{i o} K k, K l, k \preccurlyeq l, E\langle k, \bar{s}\rangle, A^{k}(\bar{s}) \Rightarrow A^{l}(\bar{s}) .
$$

\section{Lemma 3}

$$
\begin{gathered}
\vdash_{i o} \Rightarrow\left(A _ { 1 } \rightarrow \left(A_{2} \rightarrow \ldots\left(A_{n-1} \rightarrow\left(A_{n} \rightarrow B\right) \ldots\right)^{k} \leftrightarrow \forall l \succcurlyeq k\left(\bigwedge_{i} A_{i}^{l} \rightarrow B^{l}\right) .\right.\right. \\
\vdash_{i o} \Rightarrow(\forall \bar{x}(A \rightarrow B))^{k} \leftrightarrow \forall l \succcurlyeq k \forall \bar{x}\left(E\langle l, \bar{x}\rangle \wedge A^{l} \rightarrow B^{l}\right),
\end{gathered}
$$

where all free variables in $A$ and $B$ are among $\bar{x}$.

Proof We prove the second statement for $(\forall x \forall y(A(x, y) \rightarrow B(x, y)))^{k}$. We write $A$ for $A(x, y)$, etc. Note that

$$
\begin{gathered}
(\forall x \forall y(A \rightarrow B))^{k}= \\
\forall l \succcurlyeq k \forall x\left(E\langle l, x\rangle \rightarrow \forall m \succcurlyeq l \forall y\left(E\langle m, y\rangle \rightarrow \forall n \succcurlyeq m\left(A^{n} \rightarrow B^{n}\right)\right)\right) .
\end{gathered}
$$


Thus the direction from left to right of the lemma is clear. For the other direction, suppose, reasoning in LJO, that we have $\forall l \succcurlyeq k \forall x y(E\langle l, x\rangle \wedge E\langle l, y\rangle \wedge$ $\left.A^{l} \rightarrow B^{l}\right), K l, K m, K n, k \preccurlyeq l \preccurlyeq m \preccurlyeq n, E\langle l, x\rangle, E\langle m, y\rangle$ and $A^{n}$. By the upwards persistency and preorder axioms it follows that $k \preccurlyeq n$ and $E\langle n, x\rangle$ and $E\langle n, y\rangle$. Hence $B^{n}$ follows.

We define two operations on models that correspond to the above translation on formulas, one for Kripke models and one for cpo's. Transformation $(\cdot)^{o}$ transforms a Kripke model into a cpomodel such that for $A$ in $\mathcal{L}$

$$
K, k \Vdash A \Leftrightarrow K^{o} \models A^{k}
$$

and $(\cdot)^{o}$ does the converse

$$
M \models A^{k} \Leftrightarrow M^{o}, k \Vdash A .
$$

A third translation $(\cdot)^{c d}$ is introduced that translates ipo's into cpo's that are almost equivalent to it with respect to formulas of the form $A^{k}$ :

$$
K, i \Vdash A^{k} \Leftrightarrow K^{c d} \models A^{k^{i}} .
$$

\subsection{The $(\cdot)^{o}$ translation on Kripke models}

Consider a Kripke model $K=(W, \leq, D, I)$ with root $r . K$ is a model for the full language $\mathcal{L} \cup \mathcal{L}_{o} \cup \mathcal{L}_{s}$ but not necessarily an ipomodel. The construction we present below is one that will often occur in the sequel: it is a method to build a model which domain consists of the terms in a certain language, which are then interpreted as themselves.

Pick an element $y \in D_{r}$. To avoid term clashes in what follows we assume $W$, $D_{i}$, and the languages all to be disjunct. We write $D$ for $\cup_{i} D_{i}$. $D^{o}$ is the set of all closed terms in $W \cup D \cup \mathcal{L} \cup \mathcal{L}_{s} \cup \mathcal{L}_{o} \backslash\{\iota, \epsilon\}$, and $D_{k}^{o}$ is the set of all closed terms in $\mathcal{L} \cup D_{k}$. The cpomodel $K^{o}=\left(D^{o}, J\right)$ is defined as follows.

$$
\begin{aligned}
& J(K) \quad \equiv_{d e f} W \\
& J(\iota) \quad r \\
& J(\epsilon) \quad y \\
& J(\preccurlyeq) \quad \leq \\
& J(f)(\bar{c}) \quad f(\bar{c}) \\
& J(E) \quad\left\{\langle k, c\rangle \mid k \in W, c \in D_{k}^{o}\right\} \\
& J(P) \quad\left\{\left(\left\langle k, c_{1}\right\rangle, \ldots,\left\langle k, c_{n}\right\rangle\right) \mid c_{i} \in D_{k}^{o}, k \in W, k \Vdash P \bar{c}\right\}
\end{aligned}
$$

In the definition of $J$, the $P$ is an $n$-ary predicate different from $K, E$ and $\preccurlyeq$, and $f$ is different from $\iota$ and $\epsilon$. Observe that for $c \in D_{k}^{o}$ the expression $k \Vdash P \bar{c}$ is well-defined. Also note that in the definition of $E$, the $\langle k, c\rangle$ is an element of $D^{o}$, which it should be, $E$ being a unary predicate. It is easy to see that $K^{o}$ is a cpomodel; we only treat the existence axioms. That $E\langle\iota, \epsilon\rangle$ holds in $K^{o}$ follows from the fact that $\iota$ and $\epsilon$ are interpreted as $r$ and $y$ and whence $K^{o} \models E\langle r, y\rangle$. Recall that the second existence axiom is

$$
K k, E\left\langle k, x_{1}\right\rangle, \ldots, E\left\langle k, x_{n}\right\rangle \Rightarrow E\left\langle k, f\left(x_{1}, \ldots, x_{n}\right)\right\rangle \quad(f \in \mathcal{L}) .
$$


If $K^{o} \models K k \wedge E\left\langle k, c_{1}\right\rangle \wedge \ldots \wedge E\left\langle k, c_{n}\right\rangle$, this implies that $k \in W$ and $c_{i} \in D_{k}^{o}$. But then $f\left(c_{1}, \ldots, c_{n}\right) \in D_{k}^{o}$ by the definition of $D_{k}^{o}$ and the fact that $f \in \mathcal{L}$. Hence $K^{o} \models E\left\langle k, f\left(c_{1}, \ldots, c_{n}\right)\right\rangle$ because all terms except $\iota$ and $\epsilon$ are interpreted as themselves, and these two constants do not occur in $f\left(c_{1}, \ldots, c_{n}\right)$.

Recall that $\langle k, \bar{c}\rangle$ is short for $\left\langle k, c_{1}\right\rangle, \ldots,\left\langle k, c_{n}\right\rangle$ and that we write $A^{k}(\bar{t})$ for $(A(\bar{t}))^{k}$. Observe that for all predicates $P$ and all terms $\bar{s}$, both in $\mathcal{L}$ :

$$
K^{o} \models(P \bar{s}(\bar{e}))^{k} \Leftrightarrow K^{o} \models P\langle k, \bar{s}(\bar{e})\rangle \Leftrightarrow \bar{e} \in D_{k}^{o} \wedge k \in W \wedge k \Vdash P \bar{s}(\bar{e}) .
$$

Lemma 4 For all $A$ in $\mathcal{L}, k \in W$, and $\bar{e} \in D_{k}^{o}$ :

$$
K, k \Vdash A(\bar{e}) \Leftrightarrow K^{o} \models A^{k}(\bar{e}) .
$$

Proof With formula induction. Let $k \in W$. The basic case follows from (1) and the fact that $A$ is in $\mathcal{L}$ and $\bar{e} \in D_{k}^{o}$.

For the induction step we treat implication and the quantifiers. We suppress the elements $\bar{e} \in D_{k}^{o}$. Recall that $\forall l \succcurlyeq k B$ is short for $\forall l(K l \wedge l \succcurlyeq k \rightarrow B)$.

For implication we have to show that

$$
\exists l \geq k(l \Vdash A \text { and } l \Vdash B) \Leftrightarrow K^{o} \models \exists l \succcurlyeq k\left(A^{l} \wedge \neg B^{l}\right) .
$$

This follows immediately by the induction hypothesis on $l$ and the fact that $\bar{e} \in D_{k}^{o}$ implies $\bar{e} \in D_{l}^{o}$ for $l \geq k$.

For existential quantification we have to show that

$$
\exists d \in D_{k} K, k \Vdash B d \Leftrightarrow K^{o} \models \exists x\left(E\langle k, x\rangle \wedge B^{k} x\right) .
$$

$\Rightarrow$ : From $k \Vdash B d$ and $d \in D_{k}$ it follows that $K^{o} \models E\langle k, d\rangle \wedge B^{k} d$ by the induction hypothesis and the definition of $E$ and $\langle$,$\rangle , and we are done.$

$\Leftarrow$ : Suppose there is a $d \in D^{o}$ such that $K^{o}=E\langle k, d\rangle \wedge B^{k} d$. From the definition of $E$ it follows that $k \in W$ and $d \in D_{k}^{o}$. By the induction hypothesis $k \Vdash B d$ follows. This implies that there is a $e \in D_{k}$, namely $I_{k}(d)$, such that $k \Vdash B e$.

For universal quantification we have to show that

$$
\forall l \geq k \forall d \in D_{l} K, l \Vdash B d \Leftrightarrow K^{o} \models \forall l \succcurlyeq k \forall x\left(E\langle l, d\rangle \rightarrow B^{l} x\right) .
$$

or, by contraposition,

$$
\left.\exists l \geq k \exists d \in D_{l} K, l \Vdash \forall B d \Leftrightarrow K^{o} \models \exists l \succcurlyeq k \exists x\left(E\langle l, d\rangle \wedge \neg B^{l} x\right)\right) .
$$

This follows from the induction hypothesis in the same way as in the above cases.

\subsection{The $(\cdot)^{o}$ translation on cpomodels}

Given a cpomodel $M=(D, J)$ the Kripke model $M^{o}=\left(W, \leq,\left\{D_{k} \mid k \in\right.\right.$ $\left.W\},\left\{I_{k} \mid k \in W\right\}\right)$ is defined as follows. For $k, l \in D$ such that $M \models K k \wedge K l$, define

$$
k \leftrightarrow l \equiv_{\text {def }} M \models k \preccurlyeq l \wedge l \preccurlyeq k .
$$


$[k]$ denotes the equivalence class of $k$.

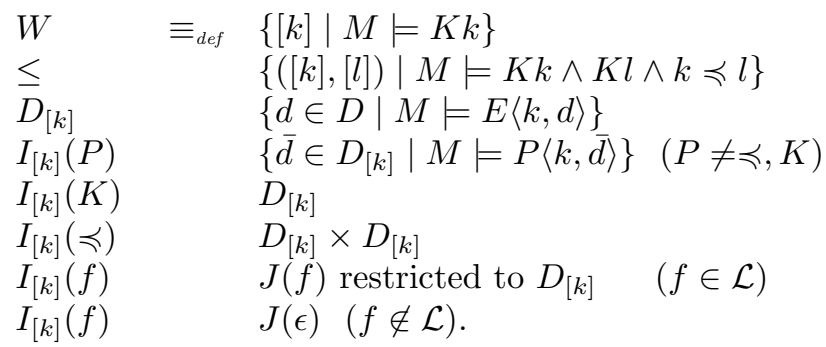

Thus all $f \notin \mathcal{L}$ are interpreted as the constant function mapping all elements to $J(\epsilon)$. Recall that we assume that all models are given in a language that has no elements in common with $\mathcal{L}, \mathcal{L}_{o}$ and $\mathcal{L}_{s}$. Thus we avoid term clashes in what follows. Note that $M^{o}$ is well-defined because of the upwards persistency axiom that holds in $M$. It is not difficult to see that $M^{o}$ is a Kripke model: $W$ and the $D_{[k]}$ are not empty because of the axioms $(\Rightarrow K \iota)$ and $(\Rightarrow E\langle\iota, \epsilon\rangle)$ in LKO, the latter implying $(K k \Rightarrow E\langle k, \epsilon\rangle)$ by the upwards persistency axiom. Clearly, $\preccurlyeq$ is a partial order on $W$, the division by the equivalence relation establishes exactly that. For all predicates except $K$ and $\preccurlyeq$ the upwards persistency in $M^{o}$ is garanteed by $A x_{u p}$. For $K$ and $\preccurlyeq$ it is trivially so. The fact that for $\bar{d} \in D_{[k]}$ also $I_{[k]}(f)(\bar{d}) \in D_{[k]}$ follows from the existence axiom $A x_{e x}$ for $f \in \mathcal{L}$, and from the fact that $M=E\langle k, \epsilon\rangle$ for $f \notin \mathcal{L}$. Observe that for predicates $P$ and terms $\bar{s}$, both in $\mathcal{L}$, we have

$$
\forall[k] \in W \forall \bar{e} \in D_{[k]}: M \models(P \bar{s}(\bar{e}))^{k} \Leftrightarrow M \models P\langle k, \bar{s}(\bar{e})\rangle \Leftrightarrow M^{o},[k] \Vdash P \bar{s}(\bar{e}) .
$$

To illustrate this: for $P(f d)$ with $f \in \mathcal{L}$, suppose $J(f)(d)=e$ and $d \in D_{[k]}$. Then $e \in D_{[k]}$ and $I_{[k]}(f)(d)=e$. Therefore indeed

$$
M=P\langle k, f d\rangle \Leftrightarrow M \models P\langle k, e\rangle \Leftrightarrow M^{o},[k] \Vdash P(e) \Leftrightarrow M^{o},[k] \Vdash P(f d) .
$$

Lemma 5 For all $A$ in $\mathcal{L}$, for all $[k] \in W$, for all $\bar{e} \in D_{[k]}$ :

$$
M \models A^{k} \bar{e} \text { iff } M^{o},[k] \Vdash A \bar{e} .
$$

Proof With formula induction. The basic case follows from (2) and the fact that $A$ is in $\mathcal{L}$ and $\bar{e} \in D_{[k]}$. For the induction step we treat implication and existential quantification. Recall that $\forall l \succcurlyeq k B$ is short for $\forall l(k \preccurlyeq l \wedge K l \rightarrow B)$. We suppress the elements $\bar{e} \in D_{[k]}$.

We have to show that

$$
\begin{gathered}
\exists[l] \in W\left([k] \leq[l] \text { and } M^{o},[l] \Vdash B \text { and } M^{o},[l] \Vdash C\right) \Leftrightarrow \\
M \models \exists l\left(K l \wedge k \preccurlyeq l \wedge B^{l} \wedge \neg C^{l}\right) .
\end{gathered}
$$

This follows from the induction hypothesis on $l$.

For existential quantification we have to show that

$$
\exists d \in D_{[k]} M^{o},[k] \Vdash B d \Leftrightarrow \exists d \in D M \models E\langle k, d\rangle \wedge B^{k} d .
$$


$\Rightarrow$ : From $d \in D_{[k]}$ it follows that $M \models E\langle k, d\rangle$, and from $[k] \Vdash B d$ it follows that $M \models B^{k} d$ by the induction hypothesis.

$\Leftarrow$ : Suppose there is a $d \in D$ such that $M \models E\langle k, d\rangle \wedge B^{k} d$. From the definition of $D_{[k]}$ it follows that $d \in D_{[k]}$, and then $[k] \Vdash B^{k} d$ follows from the induction hypothesis.

\subsection{The $(\cdot)^{c d}$ translation on ipomodels}

Given an ipomodel $K=\left(W, \leq,\left\{D_{i} \mid i \in W\right\}, I\right)$, the cpomodel $K^{c d}=(D, J)$ is defined as follows. For $i \in W$ define

$$
K^{i}=\left\{k^{i} \mid k \in D_{i}, i \Vdash K k\right\} .
$$

Here $k^{i}$ is just a symbol, namely $k$ with label $i$, and thus $k^{i}$ is distinct from $k^{j}$ whenever $i \neq j$. To avoid variable clashes below we assume $W$, the $K^{i}$, the $D_{i}$ and the languages to be disjunct from each other. The construction below slightly resembles the ()$^{o}$ translation on Kripke models. Let $D$ be the set of closed terms in $\mathcal{L} \cup \mathcal{L}_{s} \cup \mathcal{L}_{o} \cup \bigcup_{i}\left(D_{i} \cup K^{i}\right)$ and $D^{i}$ the set of closed terms in $\{\epsilon\} \cup \mathcal{L} \cup D_{i}$. The definition of $K^{c d}$ is as follows.

$$
\begin{array}{ll}
J(f)(\bar{c}) & f(\bar{c}) \\
J(P) & \left\{\left\langle k^{i}, \bar{c}\right\rangle \mid i \in W, k^{i} \in K^{i}, \bar{c} \in D^{i}, i \Vdash P\langle k, \bar{c}\rangle\right\} \quad(P \neq K, \preccurlyeq, E) \\
J(E) & \{\langle\iota, c\rangle \mid c \text { a closed term in }\{\epsilon\} \cup \mathcal{L}\} \\
& \cup\left\{\left\langle k^{i}, c\right\rangle \mid i \in W, k^{i} \in K^{i}, c \in D^{i}, i \Vdash E\langle k, c\rangle\right\} \\
J(\preccurlyeq) \quad & \{(\iota, \iota)\} \cup\left\{\left(\iota, l^{h}\right) \mid h \in W, l^{h} \in K^{h}\right\} \cup \\
& \left\{\left(k^{i}, l^{h}\right) \mid i, h \in W, k^{i} \in K^{i}, l^{h} \in K^{h}, i \leq h \Vdash k \preccurlyeq l\right\} \\
J(K) \quad & \{\iota\} \cup \bigcup_{i \in W} K^{i} .
\end{array}
$$

Lemma $6 K^{c d}$ is a cpomodel.

Proof We treat the existence and upwards persistency axioms, the preorder axioms are trivial.

For the existence axioms, that $K^{c d}$ satisfies the first one, i.e. $K^{c d} \models E\langle\iota, \epsilon\rangle$, follows by definition. For the second axiom, suppose $K^{c d}=K k \wedge E\langle k, \bar{c}\rangle$. We distinguish the cases $k=\iota$ and $k=l^{i}$. In the first case, this implies that $\bar{c}$ consists of closed terms in $\mathcal{L} \cup\{\epsilon\}$. Hence for any function $f \in \mathcal{L}, f(\bar{c})$ is a closed term in $\mathcal{L} \cup\{\epsilon\}$ as well. Since $f(\bar{c})$ is interpreted as itself in $K^{c d}$, $K^{c d} \models E\langle\iota, f(\bar{c})\rangle$ follows by definition. If $k=l^{i}$ and $K^{c d}=E\left\langle l^{i}, \bar{c}\right\rangle$, this implies that $\bar{c} \in D^{i}, i \Vdash K l$ and $i \Vdash E\langle l, \bar{c}\rangle$. Hence by the existence axiom, $i \Vdash E\langle l, f(\bar{c})\rangle$ for any $f \in \mathcal{L}$, and again, since $f(\bar{c}) \in D^{i}$ and $f(\bar{c})$ is interpreted as itself in $K^{c d}, K^{c d} \models E\left\langle l^{i}, f(\bar{c})\right\rangle$ follows by definition.

That $K^{c d}$ satisfies $A x_{u p}$ can be established as follows. Suppose $K^{c d} \models K k \wedge$ $K l \wedge k \preccurlyeq l \wedge E\langle k, \bar{c}\rangle \wedge P\langle k, \bar{c}\rangle$ for some predicate $P$ not equal to $\preccurlyeq$ and $K$. We have to show that $K^{c d} \models P\langle l, \bar{c}\rangle$. We distinguish the cases that $k=\iota$ and $k \neq \iota$.

In the latter case it follows that $k=m^{i} \in K^{i}$ and $l=n^{h} \in K^{h}$ for some $i, h \in W, m \in D_{i}, n \in D_{h}$ such that $i \leq h \Vdash m \preccurlyeq n$ and $i \Vdash K m$ and $h \Vdash K n$, 
and whence $h \Vdash K m$. Hence $K^{c d} \mid=P\langle k, \bar{c}\rangle \wedge E\langle k, \bar{c}\rangle$ implies $\bar{c} \in D^{i}$ and $K, i \Vdash P\langle m, \bar{c}\rangle \wedge E\langle m, \bar{c}\rangle$. Therefore, $\bar{c} \in D^{h}$ and $K, h \Vdash P\langle m, \bar{c}\rangle \wedge E\langle m, \bar{c}\rangle$. Thus $K, h \Vdash P\langle n, \bar{c}\rangle$, as it is an ipomodel and thus satisfies the upwards persisteny axiom. Hence $K^{c d} \models P\langle l, \bar{c}\rangle$.

In the first case, $k=\iota$ and by definition of $\preccurlyeq, l=\iota$ or $l=m^{h} \in K^{h}$ for some $h \in W$ and $m \in D_{h}$. The first case is trivial, so consider the second case. Since $K^{c d} \models P\langle\iota, \bar{c}\rangle$ it follows that $P=E$ since the other predicates do not hold on elements in $D$ of the form $\langle\iota, \bar{c}\rangle$. Therefore $K^{c d} \models E\langle\iota, c\rangle$, where $c$ is a closed term in $\mathcal{L} \cup\{\epsilon\}$. Thus $K, h \Vdash E\langle m, c\rangle$ because $K$ is an ipomodel and $K, h \Vdash K m$. This implies $K^{c d} \models E\langle l, c\rangle$.

Lemma 7 For all $A(\bar{x})$ in $\mathcal{L}, i, j \in W, \bar{c} \in D^{i}$, and all $k^{i} \in K^{i}$ :

$$
K^{c d} \models A^{k^{i}}(\bar{c}) \Leftrightarrow K, i \Vdash A^{k}(\bar{c}) .
$$

Proof Observe that $k$ and $k^{i}$ do not occur in $A$ as $\mathcal{L}$ is assumed to be disjunct from $W$, the $K^{i}$ and the $D_{i}$. For predicates $P$ different from $K, E$ and $\preccurlyeq$, we have to show that for all terms $\bar{s}(\bar{x})$ in $\mathcal{L}$

$$
K^{c d}=\left(P(\bar{s}(\bar{c}))^{k^{i}} \Leftrightarrow K, i \Vdash\left(P(\bar{s}(\bar{c}))^{k},\right.\right.
$$

that is,

$$
K^{c d} \models P\left\langle k^{i}, \bar{s}(\bar{c})\right\rangle \Leftrightarrow K, i \Vdash P\langle k, \bar{s}(\bar{c})\rangle .
$$

Note that we can assume $\bar{s}(\bar{x}) \in \mathcal{L}$ because we only have to consider atomic formulas $P(\bar{s}(\bar{x}))$ in $\mathcal{L}$. The equivalence holds because in $K^{c d}$ the term $\left\langle k^{i}, \bar{s}(\bar{c})\right\rangle$ is interpreted as itself, and then the definition of forcing for atomic formulas applies.

The induction steps for $\wedge$ and $\vee$ are easy. We treat the other cases. Note that $\bar{c} \in D^{i}$ implies $\bar{c} \in D^{h}$ for all $h \geq i$.

$\rightarrow$ : Consider $\forall l \succcurlyeq k\left(A^{l} \rightarrow B^{l}\right)$, where $A$ and $B$ are short for $A \bar{c}$ and $B \bar{e}$, where $A \bar{x}, B \bar{y} \in \mathcal{L}$ and $\bar{c} \bar{e} \in D^{i}$.

$\Rightarrow$ : Suppose $K, i \forall \forall l \succcurlyeq k\left(A^{l} \rightarrow B^{l}\right)$ for some $k^{i} \in K^{i}$, and pick $h \geq i$ with $l \in D_{h}$ and $h \Vdash K l \wedge l \succcurlyeq k \wedge A^{l}$ and $h \Vdash B^{l}$. Note that $\bar{c} \bar{e} \in D^{h}$ and $l^{h} \in K^{h}$. It follows that $K^{c d}=k^{i} \preccurlyeq l^{h} \wedge K l^{h} \wedge A^{l^{h}}$ and $K^{c d} \forall B^{l^{h}}$. Thus $K^{c d} \forall \forall \forall l \succcurlyeq k^{i}\left(A^{l} \rightarrow B^{l}\right)$.

$\Leftarrow$ : Suppose $K^{c d} \models K l \wedge l \succcurlyeq k^{i} \wedge A^{l}$, and $K^{c d} \not B^{l}$. From $K^{c d} \models K l \wedge l \succcurlyeq k^{i}$ it follows that $l=m^{h} \in K^{h}$ for some $h \in W$. Thus $m \in D_{h}$ and $h \Vdash K m$. $K^{c d} \models$ $m^{h} \succcurlyeq k^{i}$ implies $i \leq h \Vdash k \preccurlyeq m$. Thus $\bar{c} \bar{e} \in D^{h}$. By the induction hypothesis it follows that $h \Vdash A^{m}$ and $h \Downarrow B^{m}$, which implies $i \forall \forall l \succcurlyeq k\left(A^{l} \rightarrow B^{l}\right)$.

$\exists$ : Consider $\exists x\left(E\langle k, x\rangle \wedge A^{k} x\right)$, where $A x$ is short for $A x \bar{c}$, where $A x \bar{y} \in \mathcal{L}$ and $\bar{c} \in D^{i}$.

$\Rightarrow$ : Suppose there exists a $d \in D$ such that $K^{c d} \models E\left\langle k^{i}, d\right\rangle \wedge A^{k^{i}} d$. From $K^{c d} \models E\left\langle k^{i}, d\right\rangle$ it follows that $d \in D^{i}$ and $K, i \Vdash E\langle k, d\rangle$. The induction hypothesis gives $K, i \Vdash A^{k} d$. Hence there exists a $e \in D_{i}$ such that $K, i \Vdash A^{k} e$, namely $e=I_{i}(d)$. 
$\Leftarrow$ : Suppose $K, i \Vdash E\langle k, d\rangle \wedge A^{k} d$ for some $d \in D_{i}$. Clearly, $d \bar{c} \in D^{i}$. Hence the induction hypothesis implies $K^{c d}=E\left\langle k^{i}, d\right\rangle \wedge A^{k^{i}} d$.

$\forall:$ Consider $\forall l \succcurlyeq k \forall x\left(E\langle l, x\rangle \rightarrow A^{l} x\right)$, where $A x$ is short for $A x \bar{c}$, where $A x \bar{y} \in \mathcal{L}$ and $\bar{c} \in D^{i}$.

$\Rightarrow$ : Suppose $K, i \Downarrow \forall l \succcurlyeq k \forall x\left(E(l, x) \rightarrow A^{l} x\right)$ and pick a $h \geq i$ such that for some $l, d \in D_{h}, h \Vdash K l \wedge l \succcurlyeq k \wedge E\langle l, d\rangle$ and $h \Downarrow A^{l} d$. Since $i \leq h, d \bar{c} \in D^{h}$. Thus also $d \bar{c} \in D$, and $l^{h} \in K^{h}$. Thus by the induction hypothesis and the definition of $\preccurlyeq, K$ and $E, K^{c d} \models K l^{h} \wedge k^{i} \preccurlyeq l^{h} \wedge E\left\langle l^{h}, d\right\rangle$ and $K^{c d} \not \models A^{l^{h}} d$. Therefore, $K^{c d} \not \forall \forall l \succcurlyeq k^{i} \forall x\left(E\langle l, x\rangle \rightarrow A^{l} x\right)$.

$\Leftarrow$ : Suppose that for some $l, d \in D, K^{c d} \models K l \wedge l \succcurlyeq k^{i} \wedge E\langle l, d\rangle$ and $K^{c d} \not \models A^{l} d$. From $K^{c d} \models K l \wedge l \succcurlyeq k^{i}$ it follows that $l=m^{h} \in K^{h}$ for some $h \in W$ and $m \in D_{h}$, and $h \Vdash K m$ and $i \leq h \Vdash k \preccurlyeq m$. Whence $\bar{c} \in D^{h}$. From $K^{c d} \models E\left\langle m^{h}, d\right\rangle$ it follows that $d \in D^{h}$ and $h \Vdash E\langle m, d\rangle$. Hence $h \Downarrow A^{m} d$ by the induction hypothesis, which implies $K, i \| \forall l \succcurlyeq k \forall x\left(E\langle l, x\rangle \rightarrow A^{l} x\right)$.

\section{Orderization}

In this section we define the notion of orderization that we consider to be an alternative to Skolemization in constructive settings. Recall that expressions $K l$ are often supressed in formulas: e.g. $(\forall l \succcurlyeq k A(l))^{S}$ is an abbreviation of $\left(\forall l(K l \wedge k \succcurlyeq l \rightarrow A(l))^{S}\right.$, and whence equals $K l \wedge k \succcurlyeq l \rightarrow A^{S}(k)$. Recall that $A^{S}$ and $S^{S}$ denote the Skolemization of a formula $A$ and a sequent $S$, see the introduction. Note that the two $S$ 's in $S^{S}$ have a different meaning.

The orderization $A^{o}$ of a formula $A$ is a formula $\left(A^{k}\right)^{S}$, where all the variables introduced in the translation from $A$ to $A^{k}$ belong to $\mathcal{L}_{s}$. In particular, $k \in \mathcal{L}_{s}$. As is the case for Skolemization, the orderization of a formula is unique up to the renaming of variables, constants, and function symbols. The orderization $S^{o}$ of a sequent $S=(\Gamma \Rightarrow \Delta)$ is the sequent $K k,\left(\Gamma^{k}\right)^{H} \Rightarrow\left(\Delta^{k}\right)^{S}$. Thus $(I(S))^{k}$ is not equal to $I\left(S^{k}\right)$.

Example 2 Here follow some examples. For functions $f$ and arguments $d$ we write $f d$ for $f(d)$. Recall that $A\langle k, \bar{x}\rangle$ is short for $A\left(\left\langle k, x_{1}\right\rangle, \ldots,\left\langle k, x_{n}\right\rangle\right)$.

1. $(P x \rightarrow Q(f x))^{o}=$ $(\forall l \succcurlyeq k(P\langle l, x\rangle \rightarrow Q\langle l, f x\rangle))^{S} \equiv$ $K l \wedge l \succcurlyeq k \wedge P\langle l, x\rangle \rightarrow Q\langle l, f x\rangle$.

2. $(\exists x \forall y Q(x, y))^{o}=$ $(\exists x(E\langle k, x\rangle \wedge \forall l \succcurlyeq k \forall y(E\langle l, y\rangle \rightarrow Q\langle l, x, y\rangle)))^{S}=$ $\exists x(E(k, x) \wedge(K(h x) \wedge h x \succcurlyeq k \wedge E\langle h x, f x\rangle \rightarrow Q\langle h x, x, f x\rangle))$.

3. $(\exists x P x \Rightarrow Q)^{o}=$

$K k,(\exists x(E\langle k, x\rangle \wedge P\langle k, x\rangle))^{H} \Rightarrow Q^{S}=$

$K k, E\langle k, c\rangle \wedge P\langle k, c\rangle \Rightarrow Q \equiv$

$K k, E\langle k, c\rangle, P\langle k, c\rangle \Rightarrow Q$. 
4. $(\neg \neg A)^{o} \equiv K l \wedge l \succcurlyeq k \rightarrow \neg \neg \exists m \succcurlyeq l\left(A^{m}\right)^{o}$.

5. $(\neg \neg \forall x(P x \vee \neg P x))^{o} \equiv K l \wedge l \succcurlyeq k \rightarrow$

$\neg \neg \exists m \succcurlyeq l(K(h m) \wedge h m \succcurlyeq m \wedge E\langle h m, f m\rangle \rightarrow$

$(P\langle h m, f m\rangle \vee(K(g m) \wedge g m \succcurlyeq h m \rightarrow \neg P\langle g m, f m\rangle)))$.

Observe that, like standard Skolemization, equivalent formulas do not necessarily have equivalent orderizations. For example,

$$
\begin{array}{r}
(\neg \exists x P x)^{o} \equiv K l \wedge l \succcurlyeq k \rightarrow \neg(E\langle l, c\rangle \wedge P\langle l, c\rangle), \\
(\forall x \neg P x)^{o} \equiv K l \wedge l \succcurlyeq k \wedge E\langle l, c\rangle \wedge K m \wedge m \succcurlyeq l \rightarrow \neg P\langle m, c\rangle .
\end{array}
$$

Thus in many cases the orderization of a formula can simplified considerably by considering an equivalent form.

\subsection{Soundness and completeness of orderization}

From now on $S$ always denotes a closed sequent. Note that $S^{k}$ is not closed as it contains one free term, $k$. It is well-known (folklore) that

Lemma 8 For any theory $\mathcal{T}: \mathcal{T} \vdash_{\mathrm{LK}} S \Leftrightarrow \mathcal{T} \vdash_{\mathrm{LK}} S^{S}$.

Therefore,

Lemma 9 For any theory $\mathcal{T}$, for all $S: \mathcal{T} \vdash_{c o} S \Leftrightarrow \mathcal{T} \vdash_{c o} S^{S}$.

Lemma 10 For $k \in \mathcal{L}_{s}$, for all theories $\mathcal{T}$ in $\mathcal{L}: \mathcal{T} \vdash_{c o} S^{k} \Leftrightarrow T \vdash_{c o} S^{o}$.

Next we turn to the intuitionistic theories.

Lemma 11 For $S$ in $\mathcal{L}, k \in \mathcal{L}_{s}: \vdash_{\mathrm{LJ}} S \Leftrightarrow \vdash_{c o} S^{k}$.

Proof We prove the equivalence by contraposition.

$\Leftarrow$ : Assume $\forall$ LJ $S$ and let $S=(\Gamma \Rightarrow \Delta)$. Thus there is a Kripke model $K$ that forces $\wedge \Gamma$ and refutes $\bigvee \Delta$ at a node that we call $k$. Then the cpomodel $K^{o}$ satisfies $K k$ and $\wedge \Gamma^{k}$ and refutes $\bigvee \Delta^{k}$ by Lemma 4 . This implies that $\nvdash_{c o} S^{k}$ by the completeness of LKO with respect to cpomodels.

$\Rightarrow$ : Assume $\forall_{c o} S^{k}$ and let $S=(\Gamma \Rightarrow \Delta)$, and whence $S^{k}=\left(K k, \Gamma^{k} \Rightarrow \Delta^{k}\right)$. Thus there is a cpomodel $M$ that satisfies $K k$ and $\wedge \Gamma^{k}$ and refutes $\bigvee \Delta^{k}$. Then the Kripke model $M^{o}$ forces $\bigwedge \Gamma$ and refutes $\bigvee \Delta$ at $[k]$ by Lemma 5 . Thus $\forall_{L J} S$ by the completeness of LJ with respect to Kripke models.

Lemma 12 For $S$ in $\mathcal{L}, k \in \mathcal{L}_{s}: \vdash_{i o} S^{k} \Leftrightarrow \vdash_{c o} S^{k}$. 
Proof Note that this lemma does not imply that LKO $\subseteq$ LJO, which is definitely not the case: even $K k, E\langle k, x\rangle \Rightarrow P\langle k, x\rangle, \neg P\langle k, x\rangle$ is not derivable in LJO.

The direction from left to right of the lemma follows from the fact that LJO $\subseteq$ LKO. For the other direction, suppose $\forall_{i o} S^{k}$ for a closed sequent $S$. Let $S=(\Gamma \Rightarrow \Delta)$, and thus $S^{k}=\left(K k, \Gamma^{k} \Rightarrow \Delta^{k}\right)$. Hence there is an ipomodel $K$ that refutes $S^{k}$, i.e. such that $K, i \Vdash K k \wedge \wedge \Gamma^{k}$ and $K, i \Downarrow \bigvee \Delta^{k}$ for some $i$. By Lemma $7, K^{c d} \not \models \bigvee \Delta^{k_{i}}$ and $K^{c d} \models K k^{i} \wedge \wedge \Gamma^{k_{i}}$. Thus $K^{c d}$ refutes $S^{k_{i}}$. But $S^{k^{i}}$ is just a renaming of $S^{k}$, and thus the fact that $K^{c d}$ is a cpomodel, Lemma 6 , gives $\nvdash_{c o} S^{k}$.

Theorem 3 For $S$ in $\mathcal{L}, k \in \mathcal{L}_{s}$ :

$$
\vdash_{\mathrm{LJ}} S \Leftrightarrow \vdash_{i o} S \Leftrightarrow \vdash_{i o} S^{k} \Leftrightarrow \vdash_{c o} S^{k} \Leftrightarrow \vdash_{c o} S^{o} \Leftrightarrow \vdash_{i o} S^{o} .
$$

Proof By the previous lemma's and Corollary 1 it suffices to show that

$$
\vdash_{c o} S^{o} \Rightarrow \vdash_{i o} S^{o} \text {. }
$$

This follows from the fact that $\vdash_{c o} S^{o}$ implies $\vdash_{c o} S^{k}$, which implies $\vdash_{i o} S^{k}$, by the previous lemma. Since clearly $\vdash_{i o} A \Rightarrow A^{S}, \vdash_{i o} S^{o}$ follows.

Example 3 These results imply that e.g. the formula $(\forall x \neg \neg(A x \vee \neg A x))^{o}$ is derivable in LJO, while $(\neg \neg \forall x(A x \vee \neg A x))^{o}$ is not. (These formulas have been unwinded in Example 2.) Using the completeness theorems it is not difficult to give a semantical proof of this fact.

\section{Orderization in theories}

Given a theory $\mathcal{T}$ in $\mathcal{L}$, we denote by $\mathcal{T}^{k}$ the theory axiomatized by the sequents $K l, l \succcurlyeq k, E\langle l, \bar{x}\rangle, \Gamma^{l} \Rightarrow \Delta^{l}$, for every axiom $\Gamma \Rightarrow \Delta$ of $\mathcal{T}$, where $\bar{x}$ are all the free variables in $\Gamma \Delta$. Recall that $E\langle k, \bar{x}\rangle$ is short for $E\left\langle k, x_{1}\right\rangle, \ldots, E\left\langle k, x_{n}\right\rangle$. Given a sequent $K l, l \succcurlyeq k, E\langle l, \bar{x}\rangle, \Gamma^{l} \Rightarrow \Delta^{l}$, we call $K l, l \succcurlyeq k, E\langle l, \bar{x}\rangle$ the standard part of the sequent. In what follows $S$ will always be a closed sequent.

Remark 1 Recall that given a set of axioms the corresponding theory consists of all those sentences derivable from the universal closure of the axioms. Here the situation slightly changes. $\mathcal{T}^{k}$ consists of all those sentences that are derivable from the universal closure of the axioms of $\mathcal{T}^{k}$, where $k$ remains free. Therefore, for an axiom $\Gamma \Rightarrow \Delta$ of $\mathcal{T}$ with free variables $\bar{x}$, the axiom $K l, l \succcurlyeq k, E\langle l, \bar{x}\rangle, \Gamma^{l} \Rightarrow$ $\Delta^{l}$ in $\mathcal{T}^{k}$ is the open variant of the axiom $\Rightarrow \forall l \succcurlyeq k \forall \bar{x}\left(E\langle l, \bar{x}\rangle \wedge \wedge \Gamma^{l} \rightarrow \bigvee \Delta^{l}\right)$, which by Lemma 3 is equivalent to $\Rightarrow(\forall \bar{x}(\bigwedge \Gamma \rightarrow \bigvee \Delta))^{k}$ (recall that in this context $\forall l \succcurlyeq k \ldots$ is short for $\forall l \succcurlyeq k(K l \rightarrow \ldots)$. Thus the axioms of $\mathcal{T}^{k}$ are of the form $\Rightarrow A^{k}$.

Sometimes it is convenient to abbreviate the axioms of $\mathcal{T}^{k}$ by leaving out the standard parts and by replacing $l$ by $k$, but we will always say so when we 
do this. Thus in these cases, when we write $\Gamma^{k} \Rightarrow \Delta^{k}$ for an axiom in $\mathcal{T}^{k}$ it stands for the axiom $K l, l \succcurlyeq k, E\langle l, \bar{x}\rangle, \Gamma^{l} \Rightarrow \Delta^{l}$, which again stands for $\Rightarrow \forall l \succcurlyeq k \forall \bar{x}\left(E\langle l, \bar{x}\rangle \wedge \wedge \Gamma^{l} \rightarrow \bigvee \Delta^{l}\right)$.

As is the case for Skolemization, different axiomatizations $\mathcal{T}$ of the same theory can lead to different $\mathcal{T}^{k}$. For different symbols $k$ and $l$ the theories $\mathcal{T}^{k}$ and $\mathcal{T}^{l}$ are distinct, as in the first one $k$ is free and in the latter $l$ is. Of course, they are equal up to the renaming of the variables.

Theorem 4 For every theory $\mathcal{T}$ in $\mathcal{L}, S$ in $\mathcal{L}, k \in \mathcal{L}_{s}$ :

$\mathcal{T} \vdash_{\mathrm{LJ}} S \Leftrightarrow \mathcal{T} \vdash_{i o} S \Leftrightarrow \mathcal{T}^{k} \vdash_{i o} S^{k} \Leftrightarrow \mathcal{T}^{k} \vdash_{i o} S^{o} \Leftrightarrow \mathcal{T}^{k} \vdash_{c o} S^{k} \Leftrightarrow \mathcal{T}^{k} \vdash_{c o} S^{o}$.

Proof All these equivalences follow from the corresponding statements above for LJO pure. The first equivalence is Corollary 1. As an example, we prove that

$$
\mathcal{T} \vdash_{\mathrm{LJ}} S \Leftrightarrow \mathcal{T}^{k} \vdash_{c o} S^{k} .
$$

$\Rightarrow$ : Assume $\mathcal{T} \nvdash_{L J} S$ and let $S=(\Gamma \Rightarrow \Delta)$. Hence there is a Kripke model $K$ of $\mathcal{T}$ that forces $\Lambda \Gamma$ and refurtes $\bigvee \Delta$ at a node that we call $k$. Then the cpomodel $K^{o}$ satisfies $\Lambda \Gamma^{k}$ and $K k$ and refutes $\bigvee \Delta^{k}$ by Lemma 4 . Since $K$ is a Kripke model of $\mathcal{T}$ every node in it satisfies $\forall \bar{x}(\bigwedge \Pi \rightarrow \bigvee \Theta)$ for every axiom $(\Pi \rightarrow \Theta)$ of $\mathcal{T}$ with free variables $\bar{x}$. Again by Lemma 4 , this implies that $K^{o}$ satisfies $(\forall \bar{x}(\bigwedge \Pi \rightarrow \bigvee \Theta))^{k}$. Therefore it is a model of $\mathcal{T}^{k}$.

$\Leftarrow$ : Assume $\mathcal{T}^{k} \forall_{c o} S^{k}$ and let $S=(\Gamma \Rightarrow \Delta)$. Thus $S^{k}=\left(K k, \Gamma^{k} \Rightarrow \Delta^{k}\right)$. There is a cpomodel $M$ of $\mathcal{T}^{k}$ that satisfies $K k$ and $\Lambda \Gamma^{k}$ and refutes $\bigvee \Delta^{k}$. Hence by Lemma 5 the Kripke model $M^{o}$ forces $\Lambda \Gamma$ and refutes $\bigvee \Delta$ at node $[k]$. Since $M$ is a cpomodel of $\mathcal{T}^{k}$ it satisfies $(\forall \bar{x}(\bigwedge \Pi \rightarrow \bigvee \Theta))^{k}$ for all axioms $(\Pi \Rightarrow \Theta)$ with free variables $\bar{x}$. By Lemma 5 this implies that $M^{o}$ forces $\forall \bar{x}(\bigwedge \Pi \rightarrow \bigvee \Theta)$ at $[k]$. Thus there is a Kripke model of $\mathcal{T}$ that refutes $S$, namely the restriction of $M^{o}$ to the cone above $[k]$. Hence $\mathcal{T} \forall\llcorner J S$.

Similar reasoning applies to the equivalence $\mathcal{T}^{k} \vdash_{i o} S^{k} \Leftrightarrow \mathcal{T}^{k} \vdash_{c o} S^{k}$, via the ( $)^{c d}$ construction: for $S=(\Gamma \Rightarrow \Delta)$, consider an ipomodel $K$ of $\mathcal{T}^{k}$ that satisfies $K k \wedge \wedge \Gamma^{k}$ and that refutes $\bigvee \Delta^{k}$, say at node $i$. By Lemma $7, K^{c d}$ is a cpomodel of $\mathcal{T}^{k^{i}}$ that refutes $S^{k^{i}}$. Therefore, $\mathcal{T}^{k} \nvdash_{c o} S^{k}$.

The equivalence $\mathcal{T}^{k} \vdash_{i o} S^{o} \Leftrightarrow \mathcal{T}^{k} \vdash_{c o} S^{o}$ has the same proof as for LJO pure, using the fact that Skolemization is also complete in case only some but not necessarily all the strong quantifiers in a formula are replaced.

The remainder of this section is devoted to analogues of this theorem in which the theory $\mathcal{T}^{k}$ is replaced by theories closer to $\mathcal{T}$ itself. The following two lemmas give conditions under which such theorems might be obtained.

Lemma 13 For theories $\mathcal{T}$ in $\mathcal{L}, S$ in $\mathcal{L}, k \in \mathcal{L}_{s}$ :

$$
\begin{aligned}
& \mathcal{T} \vdash_{\mathrm{LJ}} A \Leftrightarrow \mathcal{T} \vdash_{i o} S \\
& \mathcal{T} \vdash_{i o} S^{k} \Rightarrow \mathcal{T} \vdash_{i o} S^{o} \Rightarrow \mathcal{T} \vdash_{c o} S^{o} \Leftrightarrow \mathcal{T} \vdash_{c o} S^{k} .
\end{aligned}
$$


Corollary 2 For theories $\mathcal{T}$ and $\mathcal{T}^{\prime}$ in $\mathcal{L}, S$ in $\mathcal{L}, k \in \mathcal{L}_{s}$, if

$$
\mathcal{T} \vdash_{i o} S \Leftrightarrow \mathcal{T}^{\prime} \vdash_{i o} S^{k} \text { and } \mathcal{T}^{\prime} \vdash_{c o} S^{k} \Rightarrow \mathcal{T}^{\prime} \vdash_{i o} S^{k},
$$

then

$\mathcal{T} \vdash_{\text {LJ }} A \Leftrightarrow \mathcal{T} \vdash_{i o} S \Leftrightarrow \mathcal{T}^{\prime} \vdash_{i o} S^{k} \Leftrightarrow \mathcal{T}^{\prime} \vdash_{i o} S^{o} \Leftrightarrow \mathcal{T}^{\prime} \vdash_{c o} S^{o} \Leftrightarrow \mathcal{T}^{\prime} \vdash_{c o} S^{k}$.

\subsection{Equality}

The first adaption of $\mathcal{T}^{k}$ concerns the equality axioms of $\mathcal{T}$, in case it contains these. Note that in this case $=$ belongs to $\mathcal{L}$, which is the language of $\mathcal{T}$. We will see that the ()$^{k}$ translations of the equality axioms can be replaced by the axioms itself.

Let $\mathcal{T}$ be a theory in $\mathcal{L}$ that includes the equality axioms

$$
\begin{aligned}
A x_{e q} \quad \equiv_{d e f} & \Rightarrow x=x \\
& x=y \Rightarrow y=x, \\
& x=y, y=z \Rightarrow x=z \\
& \bar{x}=\bar{y}, P(\bar{x}) \Rightarrow P(\bar{y}) \quad(P \text { a predicate in } \mathcal{L}) \\
& \bar{x}=\bar{y} \Rightarrow f(\bar{x})=f(\bar{y}) \quad(f \text { a function in } \mathcal{L})
\end{aligned}
$$

We denote by $\mathcal{T}_{k}$ the theory in $\mathcal{L} \cup \mathcal{L}_{o}$ consisting of the axioms of $\mathcal{T}$ plus

$$
A x_{k} \quad K l, l \succcurlyeq k, E\langle l, \bar{x} \bar{y}\rangle,\langle l, \bar{x}\rangle=\langle l, \bar{y}\rangle \Rightarrow\langle l, f(\bar{x})\rangle=\langle l, f(\bar{y})\rangle
$$

$(f$ a function in $\mathcal{L})$

Observe that this axiom is part of $A x_{e q}^{k}$, which is given by the following axioms, where we have left out the standard parts as explained at the beginning of Section 7. (Recall that $\langle k, \bar{x}\rangle=\langle k, \bar{y}\rangle$ is short for $\left\{\left\langle k, x_{i}\right\rangle=\left\langle k, y_{i}\right\rangle \mid i \leq n\right\}$.)

$$
\begin{aligned}
A x_{e q}^{k} \quad \equiv_{\text {def }} & \Rightarrow\langle k, x\rangle=\langle k, x\rangle \\
& \langle k, x\rangle=\langle k, y\rangle \Rightarrow\langle k, y\rangle=\langle k, x\rangle \\
& \langle k, x\rangle=\langle k, y\rangle,\langle k, y\rangle=\langle k, z\rangle \Rightarrow\langle k, x\rangle=\langle k, z\rangle \\
& \langle k, \bar{x}\rangle=\langle k, \bar{y}\rangle, P(\langle k, \bar{x}\rangle) \Rightarrow P(\langle k, \bar{y}\rangle) \quad(P \text { a predicate in } \mathcal{L}) \\
& \langle k, \bar{x}\rangle=\langle k, \bar{y}\rangle \Rightarrow\langle k, f(\bar{x})\rangle=\langle k, f(\bar{y})\rangle \quad(f \text { a function in } \mathcal{L})
\end{aligned}
$$

Compare these axioms to equality axioms in type structures, where there exist $={ }_{\sigma}$ for any type $\sigma$.

Lemma 14 For $\vdash \in\left\{\vdash_{i o}, \vdash_{c o}\right\}$, for $\mathcal{T}$ in $\mathcal{L}, S$ in $\mathcal{L}, k \in \mathcal{L}_{s}: \mathcal{T} \vdash S \Leftrightarrow \mathcal{T}_{k} \vdash S$.

Proof We treat the case $\vdash_{c o}$, the other one being similar. The direction from left to right is clear. For the other direction, let $M=(D, I)$ be a cpomodel of $\mathcal{T}$ that refutes $S$. We build a cpomodel $N=(D, J)$ of $\mathcal{T}_{k}$ that refutes $S$ as follows. 
Fix a $d \in D$. $N$ is $M$ except for the interpetation of $E$ which holds only on $d$, and the interpretation of $\langle$,$\rangle , which maps all pairs to d$. Clearly, for all $B$ in $\mathcal{L}$ and $\bar{c} \in D$ :

$$
N \models B(\bar{c}) \Leftrightarrow M \models B(\bar{c}) .
$$

From this it follows that $N$ refutes $S$ and is a model of $\mathcal{T}$. That it is a model of $A x_{k}$ follows immediately from the interpretation of $E$ and $\langle$,$\rangle in N$. That $N$ is a cpomodel is also easy to verify.

We will return to theories that contain equality in the section on applications below.

\subsection{Atomic theories}

In the next section we will apply the results obtained so far to the intuitionistic theories of equality and of groups. In the light of Theorem 4 , if a theory $\mathcal{T}$ does not contain many implications or quantifiers it will be close to $\mathcal{T}^{k}$, often implying it. The theories of equality, groups, apartness and linear orders are examples of this. The reason that we have included the axioms $\Rightarrow K \iota$ and $\Rightarrow E\langle\iota, \epsilon\rangle$ instead of $\Rightarrow \exists x K x$ and $K k \Rightarrow \exists x E\langle k, x\rangle$ is that otherwise LJO would be a theory which axioms contain quantifiers. Due to lack of space we will in this paper only discuss atomic theories, which are theories in which the antecedent of every axiom consists of formulas of the form $P(\bar{x})$ for some predicates $P$ and free variables $\bar{x}$, and the succedent is an atomic formula, so of the form $Q(\bar{t})$ for some terms $\bar{t}$ and predicate $Q$. Observe that atomicity of a theory is a stricter requirement than having the axiom sequents consist of atomic formulas only. As before $S$ ranges over closed sequents.

Lemma 15 For atomic theories $\mathcal{T}$ in $\mathcal{L}, S$ in $\mathcal{L}, k \in \mathcal{L}_{s}$ :

$$
\mathcal{T}_{k} \vdash_{c o} S^{k} \Rightarrow \mathcal{T}^{k} \vdash_{c o} S^{k}
$$

Proof Arguing contrapositively, assume $M=(D, I)$ is a cpomodel of $\mathcal{T}^{k}$ that refutes $S^{k}=(\Gamma \Rightarrow \Delta)^{k}$. Observe that this fixes $k$ and that $M=K k$, since $M$ satisfies $K k, \bigwedge \Gamma^{k}$ and refutes $\bigvee \Delta^{k}$. We construct a cpomodel $N=\left(D^{\prime}, J\right)$ of $\mathcal{T}_{k}$ that refutes $S^{k}$ as well.

$$
\begin{aligned}
& D^{\prime} \equiv_{\text {def }} \quad\left\{t \mid t \text { is a closed term in } \mathcal{L} \cup \mathcal{L}_{s} \cup \mathcal{L}_{o} \cup D\right\} \\
& J(f)(\bar{d}) \quad f(\bar{d}) \quad(f \text { a function }) \\
& J(R) \quad I(R) \quad(R=K, \preccurlyeq, E)
\end{aligned}
$$

Clearly, in $N$ every closed term is interpreted as itself. $J(R)=I(R)$ should be read as $J(R)=\left\{\bar{t} \in D^{\prime}|M|=R \bar{t}\right\}$. Observe that because of this definition it follows that $N$ is a model of $A x_{p o}$ and $A x_{e x} . A x_{u p}$ is satisfied in case the predicate is $E$. That all of $A x_{u p}$ holds in $N$ will be shown below.

Validity in $N$ on atomic formulas in $\mathcal{L}$ is defined inductively via the relations $\models_{i}$. Let the axioms of $\mathcal{T}$ outside LJO be $\left\{S_{i} \mid i \in \mathcal{I}\right\}$ for some set 
$\mathcal{I}$. Because $\mathcal{T}$ is atomic we can suppose all these sequents to be of the form $S_{i}=P_{i 1}(\bar{x}), \ldots, P_{i m_{i}}(\bar{x}) \Rightarrow P_{i}\left(\bar{t}_{i}(\bar{x})\right)$, where the $P_{i j}$ and $P_{i}$ are predicates in $\mathcal{L}$ and the $\bar{t}_{i}(\bar{x})$ are terms in $\mathcal{L}$. For $P$ in $\mathcal{L}$ we define:

$$
\begin{aligned}
& N \models_{0} P \quad \equiv_{\text {def }} \quad M \models P \text {, for } P \text { without free variables } \\
& N \models{ }_{0} P(\bar{c}) \quad \exists l, \bar{e} \in D^{\prime}:\langle l, \bar{e}\rangle=\bar{c} \text { and } M=k \preccurlyeq l \wedge P(\bar{c}) \\
& N \models_{n+1} P(\bar{c}) \quad N=_{n} P(\bar{c}) \text {, or } \\
& \exists i \in \mathcal{I} \exists \bar{e} \in D^{\prime}: m_{i} \geq 1 \text { and } P(\bar{c})=P_{i}\left(\bar{t}_{i}(\bar{e})\right) \text { and } \\
& N \models P(\bar{c}) \quad \exists n \bar{N} \models_{n} P(\bar{c}) .
\end{aligned}
$$

Observe that in this definition $\langle l, \bar{e}\rangle=\bar{c}$ means that $\bar{c}$ and $\langle l, \bar{e}\rangle$ are literally the same string of symbols, and it therefore is a stronger requirement than merely $M \models\langle l, \bar{e}\rangle=\bar{c}$. A similar remark applies to $P(\bar{c})=P_{i}\left(\bar{t}_{i}(\bar{e})\right)$.

First we show that for all $B(\bar{x})$ in $\mathcal{L}$, and all $l, \bar{e} \in D^{\prime}$ such that $M \models K l \wedge k \preccurlyeq$ $l \wedge E\langle l, \bar{e}\rangle$ :

$$
N=B(\bar{e})^{l} \Leftrightarrow M \models B(\bar{e})^{l} .
$$

Note that since all terms in $D^{\prime}$ are closed terms in $\mathcal{L} \cup \mathcal{L}_{s} \cup \mathcal{L}_{o} \cup D$ the expression $M \models B(\bar{e})^{l}$ is well-defined.

For the basic case of $(3)$ we have to show that for all $P(\bar{x})$ in $\mathcal{L}$ :

$$
N \models P\langle l, \bar{e}\rangle \Leftrightarrow M \models P\langle l, \bar{e}\rangle .
$$

The direction from right to left follows immediately from the definition of $\models_{0}$ and the interpretation of terms in $N$. The other direction is proved by induction on $\models_{i}$. The case $n=0$ is trivial. Suppose $N \models_{n+1} P\langle l, \bar{c}\rangle$ for some $l$ such that $M \models K l \wedge k \preccurlyeq l \wedge E\langle l, \bar{c}\rangle$. Hence for some $i \in \mathcal{I}, P\langle l, \bar{c}\rangle=P_{i}\left(\bar{t}_{i}(\bar{e})\right)$ and $N \models_{n} P_{i h}(\bar{e})$ for all $h \leq m_{i}$. Thus $P=P_{i}$ and $\langle l, \bar{c}\rangle=\bar{t}_{i}(\bar{e})$. Since $P_{i}\left(\bar{t}_{i}(\bar{x})\right)$ is in $\mathcal{L}$, it follows that $\bar{t}_{i}(\bar{x})$ is in $\mathcal{L}$, and whence $\bar{t}_{i}(\bar{x})=\bar{x}$, and thus $\bar{t}_{i}(\bar{e})=\bar{e}=\langle l, \bar{c}\rangle$, since $\langle$,$\rangle is not in \mathcal{L}$. By the induction hypothesis $M \models P_{i h}\langle l, \bar{c}\rangle$. Using the fact that

$$
\Rightarrow \forall l \succcurlyeq k \forall \bar{x}\left(E\langle l, \bar{x}\rangle \wedge P_{i 1}\langle l, \bar{x}\rangle \wedge \ldots \wedge P_{i m_{i}}\langle l, \bar{x}\rangle \rightarrow P_{i}\left\langle l, \bar{t}_{i}(\bar{x})\right\rangle\right)
$$

is an axiom of $\mathcal{T}^{k}$, and hence valid in $M$, it follows that $M \models P_{i}\left\langle l, \bar{t}_{i}(\bar{c})\right\rangle$, i.e. $M \models P_{i}\langle l, \bar{c}\rangle$, that is, $M=P\langle l, \bar{c}\rangle$.

The induction steps for the connectives are straighforward. For the quantifiers, first consider $\exists x\left(E\langle l, x\rangle \wedge A^{l}(x, \bar{e})\right)$ for some $A$ in $\mathcal{L}, l, \bar{e} \in D^{\prime}$ such that $M \models$ $K l \wedge k \preccurlyeq l \wedge E\langle l, \bar{e}\rangle$. We suppress $\bar{e}$ in what follows.

$\Rightarrow$ : Suppose there is a $d \in D^{\prime}$ such that $N \models E\langle l, d\rangle \wedge A^{l}(d)$. Hence $M \models E\langle l, d\rangle$ since $E$ is interpreted in the same way in $N$ as in $M$. Therefore the induction hypothesis applies.

$\Leftarrow$ : Suppose there is a $d \in D$ such that $M \models E\langle l, d\rangle \wedge A^{l}(d)$. Again, the induction hypothesis plus the fact that $E$ is interpreted in he same way in $N$ as in $M$, implies the result.

For the universal quantifier, consider the formula $\forall m \succcurlyeq l \forall x\left(E\langle m, x\rangle \rightarrow A^{m}(x, \bar{e})\right)$ for some $A$ in $\mathcal{L}, l, \bar{e} \in D^{\prime}$ such that $M \models K l \wedge k \preccurlyeq l \wedge E\langle l, \bar{e}\rangle$. We suppress $\bar{e}$ 
in what follows.

$\Rightarrow$ : Suppose $M \forall \forall \forall m \succcurlyeq l \forall x\left(E\langle m, x\rangle \rightarrow A^{m}(x, \bar{e})\right)$. Then there are $m, d \in D$ with $M \models K m \wedge l \preccurlyeq m \wedge E\langle m, d\rangle \wedge \neg A^{m} d$. Hence $M=k \preccurlyeq m$. Thus the induction hypothesis and the interpretation of $K$, $\preccurlyeq$ and $E$ in $N$ imply $N \models K m \wedge l \preccurlyeq m \wedge E\langle m, d\rangle \wedge \neg A^{m} d$.

$\Leftarrow$ : Suppose there are $m, d \in D^{\prime}$ with $N \models K m \wedge l \preccurlyeq m \wedge E\langle m, d\rangle \wedge \neg A^{m} d$. Hence $M \models K m \wedge k \preccurlyeq m \wedge l \preccurlyeq m \wedge E\langle m, d\rangle$. The induction hypothesis gives $M \models \neg A^{m} d$.

Equation (3), the fact that $M \models K k \wedge k \preccurlyeq k$ and that $S$ is closed, imply that $N$ refutes $S^{k}$. That it satisfies $\mathcal{T}$, i.e.

$$
N \mid \forall \bar{x}\left(P_{i 1}(\bar{x}) \wedge \ldots \wedge P_{i m_{i}}(\bar{x}) \rightarrow P_{i}\left(\bar{t}_{i}(\bar{x})\right)\right)
$$

follows immdediately from the definition of $N$.

To see that $N$ is a cpomodel it only remains to be shown that it satisfies $A x_{u p}$ for $P \in \mathcal{L}$. Therefore, suppose $N \mid=K l \wedge K m \wedge l \preccurlyeq m \wedge E\langle l, \bar{c}\rangle \wedge P\langle l, \bar{c}\rangle$. We have to show that $N \models P\langle m, \bar{c}\rangle$. By equation (3) it suffices to show that $M \models k \preccurlyeq l$. We prove this with induction to $\models_{n}$. For $n=0$, i.e. if $N \models_{0} P\langle l, \bar{c}\rangle$, the statement is clear. And if $N \models{ }_{n+1} P\langle l, \bar{c}\rangle$, then for some $i, P\langle l, \bar{c}\rangle=P_{i}\left(\bar{t}_{i}(\bar{e})\right)$ and $N={ }_{n} P_{i h}(\bar{e})$ for all $h \leq m_{i}$. Thus the same reasoning as above applies: $\langle l, \bar{c}\rangle=\bar{t}_{i}(\bar{e})=\bar{e}$ since $\bar{t}_{i}(\bar{x})$ is in $\mathcal{L}$ and $\langle$,$\rangle is not. m_{i} \geq 1$ and the induction hypothesis for $N \models{ }_{n} P_{i 1}\langle l, \bar{c}\rangle$ implies that $M \models k \preccurlyeq l$.

It remains to be shown that $N$ also is a model of $A x_{k}$,

$$
\Rightarrow \forall l \succcurlyeq k \forall \bar{x} \forall \bar{y}(K l \wedge E\langle l, \bar{x} \bar{y}\rangle \wedge\langle l, \bar{x}\rangle=\langle l, \bar{y}\rangle \rightarrow\langle l, f(\bar{x})\rangle=\langle l, f(\bar{y})\rangle)
$$

$(f$ a function in $\mathcal{L}$ )

This follows from (3), using that $\langle l, \bar{x}\rangle=\langle l, \bar{y}\rangle$ and $\langle l, f(\bar{x})\rangle=\langle l, f(\bar{y})\rangle$ are of the form $B^{l}$ for some $B$ in $\mathcal{L}$ since $f$ and $=$ are both in $\mathcal{L}$, and the fact that $M$ satisfies the sequent.

The following theorem shows that $\mathcal{T}_{k}$ is an interesting theory.

Theorem 5 For every atomic theory $\mathcal{T}$ in $\mathcal{L}$ for which $\mathcal{T}_{k}$ derives $\mathcal{T}^{k}$, for $S$ in $\mathcal{L}, k \in \mathcal{L}_{s}$ :

$$
\begin{gathered}
\mathcal{T} \vdash_{\text {LJ }} S \Leftrightarrow \mathcal{T} \vdash_{i o} S \Leftrightarrow \mathcal{T}_{k} \vdash_{i o} S \Leftrightarrow \mathcal{T}_{k} \vdash_{i o} S^{k} \Leftrightarrow \\
\mathcal{T}_{k} \vdash_{\text {co }} S^{k} \Leftrightarrow \mathcal{T}_{k} \vdash_{\text {co }} S^{o} \Leftrightarrow \mathcal{T}_{k} \vdash_{i o} S^{o} .
\end{gathered}
$$

Proof By Corollary 2 it suffices to show that

$$
\mathcal{T} \vdash_{i o} S \Leftrightarrow \mathcal{T}_{k} \vdash_{i o} S^{k} \text { and } \mathcal{T}_{k} \vdash_{c o} S^{k} \Rightarrow \mathcal{T}_{k} \vdash_{i o} S^{k}
$$

These statements follow from Theorem 4 and Lemma 15, using the fact that $T_{k}$ derives $\mathcal{T}^{k}$ by assumption. 


\subsection{Herbrand's Theorem}

In the context of intuitionistic logic there is a natural analogue of Herbrand's theorem. Following [7], define a sequent $S^{\prime}$ to be a $\wedge \vee$-expansion (or a Herbrand expansion) of a sequent $S$ if every positive occurrence of an existential quantifier $Q x A(x)$ in $S$ is replaced by $\bigvee_{i=1}^{m} A\left(s_{i}\right)$ for some terms $s_{i}$, and every negative occurrence of a universal quantifier $Q x A(x)$ is replaced by $\bigwedge_{i=1}^{n} A\left(t_{i}\right)$ for some terms $t_{i}$. By considering proofs in LJ the following analogue of Herbrand's theorem is easy to prove. Again, $S$ will always denote a closed sequent.

Theorem 6 For every universal theory $\mathcal{T}$ and every sequent $S$ in $\mathcal{L}$ without strong quantifiers there exists an $\wedge \vee$-expansion $S^{\prime}$ of it such that

$$
\mathcal{T} \vdash_{\text {LJ }} S \Leftrightarrow \mathcal{T} \vdash_{\text {LJ }} S^{\prime} .
$$

Proof We treat the case that $S$ only contains one quantifier, a positive occurrence of $\exists x A x$. Consider all places in the proof where the quantifier is introduced. Because the quantifier occurs positively this can only happen in an application of right weakening or $\mathrm{R} \exists$. It cannot occur in an axiom of $\mathcal{T}$ since it is a universal theory, and it cannot appear in the axioms of LJO because these are quantifier free. Suppose there are $n$ places where $\exists x A x$ is introduced via $\mathrm{R} \exists$, and that they are of the form

$$
\mathrm{R} \exists \frac{\Gamma_{i} \Rightarrow A t_{i}}{\Gamma_{i} \Rightarrow \exists x A x}
$$

Then we transform the proof by replacing every such occurrence by

$$
\mathrm{R} \vee \frac{\Gamma_{i} \Rightarrow A t_{i}}{\Gamma_{i} \Rightarrow \bigvee_{i=1}^{n} A\left(t_{i}\right)}
$$

Finally we replace instances of RW on $\exists x A x$,

$$
\mathrm{RW} \frac{\Pi \Rightarrow}{\Pi \Rightarrow \exists x A x}
$$

by

$$
\mathrm{RW} \frac{\Pi \Rightarrow}{\Pi \Rightarrow \bigvee_{i=1}^{n} A\left(t_{i}\right)}
$$

It is not difficult to see that the result is a proof of the $\wedge \vee$-expansion of $A$ in which the $\exists x A x$ 's have been replaced by $\bigvee_{i=1}^{n} A\left(t_{i}\right)$. Observe that the side conditions on variables in $\mathrm{R} \forall$ and $\mathrm{L} \exists$ cannot be violated since $S$ does not contain strong quantifiers.

A similar proof implies the same result for LJO:

Theorem 7 For every universal theory $\mathcal{T}$, and for every sequent $S$ in $\mathcal{L}$ without strong quantifiers there exists an $\wedge \vee$-expansion $S^{\prime}$ of it such that

$$
\mathcal{T} \vdash_{i o} S \Leftrightarrow \mathcal{T} \vdash_{i o} S^{\prime}
$$


The theorem above is the reason that we included the axioms $(\Rightarrow K \iota)$ and $(\Rightarrow E\langle\iota, \epsilon\rangle)$ in LJO, since replacing them by the sequents $(\Rightarrow \exists x K x)$ and $(K k \Rightarrow$ $\exists x E\langle k, x\rangle)$ would have made LJO into a theory that is no longer quantifier free. The last theorem and Theorem 4 imply the following correspondence between predicate and propositional logic.

Corollary 3 For every theory $\mathcal{T}$ in $\mathcal{L}$ for which $\mathcal{T}^{k}$ is universal, for $S$ in $\mathcal{L}$, $k \in \mathcal{L}_{s}$ there exists a quantifier free $\wedge \vee$-expansion $S^{\prime}$ of $S^{o}$ such that

$$
\begin{aligned}
& \mathcal{T} \vdash_{\text {LJ }} S \Leftrightarrow \mathcal{T} \vdash_{i o} S \Leftrightarrow \mathcal{T}^{k} \vdash_{i o} S^{k} \Leftrightarrow \mathcal{T}^{k} \vdash_{c o} S^{k} \Leftrightarrow \\
& \mathcal{T}^{k} \vdash_{c o} S^{o} \Leftrightarrow \mathcal{T}^{k} \vdash_{i o} S^{o} \Leftrightarrow \mathcal{T}^{k} \vdash_{c o} S^{\prime} \Leftrightarrow \mathcal{T}^{k} \vdash_{i o} S^{\prime} .
\end{aligned}
$$

In particular, there exists a quantifier free $\wedge \vee$-expansion $S^{\prime}$ of $S^{o}$ such that

$\vdash_{\text {LJ }} S \Leftrightarrow \vdash_{i o} S \Leftrightarrow \vdash_{i o} S^{k} \Leftrightarrow \vdash_{c o} S^{k} \Leftrightarrow \vdash_{c o} S^{o} \Leftrightarrow \vdash_{i o} S^{o} \Leftrightarrow \vdash_{c o} S^{\prime} \Leftrightarrow \vdash_{i o} S^{\prime}$.

Observe that the $\wedge$ V-expansion of $S^{o}$ obtained via proofs of $S^{o}$ in LJO and LKO might be different in case the proofs of $S^{o}$ are distinct. However, because of the other equivalences, there is always an $\wedge \vee$-expansion for which all the equivalences hold.

Corollary 2 and Theorem 7 imply

Corollary 4 For $S$ in $\mathcal{L}, k \in \mathcal{L}_{s}$, and for all theories $\mathcal{T}$ for which there exists a universal theory $\mathcal{T}^{\prime}$ such that $\left(\mathcal{T} \vdash_{i o} S \Leftrightarrow \mathcal{T}^{\prime} \vdash_{i o} S^{k}\right)$ and $\left(\mathcal{T}^{\prime} \vdash_{c o} S^{k} \Rightarrow\right.$ $\left.\mathcal{T}^{\prime} \vdash_{i o} S^{k}\right)$, there exists a quantifier free $\wedge \vee$-expansion $S^{\prime}$ of $S^{o}$ such that

$$
\begin{gathered}
\mathcal{T} \vdash_{\mathrm{LJ}} S \Leftrightarrow \mathcal{T} \vdash_{i o} S \Leftrightarrow \mathcal{T}^{\prime} \vdash_{i o} S^{k} \Leftrightarrow \mathcal{T}^{\prime} \vdash_{c o} S^{o} \Leftrightarrow \\
\mathcal{T}^{\prime} \vdash_{c o} S^{o} \Leftrightarrow \mathcal{T}^{\prime} \vdash_{i o} S^{o} \Leftrightarrow \mathcal{T}^{\prime} \vdash_{c o} S^{\prime} \Leftrightarrow \mathcal{T}^{\prime} \vdash_{i o} S^{\prime} .
\end{gathered}
$$

Clearly, if $\mathcal{T}$ is atomic, then $\mathcal{T}_{k}$ is a universal theory. Thus by Theorem 5 we have

Corollary 5 For every atomic theory $\mathcal{T}$ in $\mathcal{L}$ for which $\mathcal{T}_{k}$ derives $\mathcal{T}^{k}$, for $S$ in $\mathcal{L}, k \in \mathcal{L}_{s}$, there exists a quantifier free $\wedge \vee$-expansion $S^{\prime}$ of $S^{o}$ such that

$$
\begin{gathered}
\mathcal{T} \vdash_{\text {LJ }} S \Leftrightarrow \mathcal{T} \vdash_{i o} S \Leftrightarrow \mathcal{T}_{k} \vdash_{i o} S \Leftrightarrow \mathcal{T}_{k} \vdash_{i o} S^{k} \Leftrightarrow \mathcal{T}_{k} \vdash_{c o} S^{o} \Leftrightarrow \\
\mathcal{T}_{k} \vdash_{c o} S^{o} \Leftrightarrow \mathcal{T}_{k} \vdash_{i o} S^{o} \Leftrightarrow \mathcal{T}_{k} \vdash_{c o} S^{\prime} \Leftrightarrow \mathcal{T}_{k} \vdash_{i o} S^{\prime} .
\end{gathered}
$$

Observe that the decidability result in Section 9 implies that for every $\wedge \vee$ expansion of a sequent of the form $S^{o}$ it is decidable whether it is derivable in one of our order calculi or not.

\section{Applications}

\subsection{The theory of equality}

For the theory of equality iEq given by the axioms $A x_{e q}$, we have seen above what the axioms of $\mathrm{iEq}^{k}$ and $\mathrm{iEq}_{k}$ are. Thus in this case $=$ belongs to $\mathcal{L}$, which 
is the language of the theory $\mathrm{iEq}$. Since $\mathrm{iEq}^{k}$ is clearly universal, Corollary 3 implies

Corollary 6 For $S$ in $\mathcal{L}, k \in \mathcal{L}_{s}$ there exists a quantifier free $\wedge \vee$-expansion $S^{\prime}$ of $S^{o}$ such that

$$
\begin{aligned}
& \mathrm{iEq} \vdash_{\mathrm{LJ}} S \Leftrightarrow \mathrm{iEq} \vdash_{i o} S \Leftrightarrow \mathrm{iEq}^{k} \vdash_{i o} S^{k} \Leftrightarrow \mathrm{iEq}^{k} \vdash_{c o} S^{k} \Leftrightarrow \\
& \mathrm{iEq}^{k} \vdash_{c o} S^{o} \Leftrightarrow \mathrm{iEq}^{k} \vdash_{i o} S^{o} \Leftrightarrow \mathrm{iEq}^{k} \vdash_{i o} S^{\prime} \Leftrightarrow \mathrm{iEq}^{k} \vdash_{c o} S^{\prime} .
\end{aligned}
$$

Clearly, $\mathrm{i} E q$ is an atomic theory and $\mathrm{iEq}_{k}$ derives $\mathrm{iEq}^{k}$. Therefore, by Corollary 5 we have

Corollary 7 For $S$ in $\mathcal{L}, k \in \mathcal{L}_{s}$ there exists a quantifier free $\wedge \vee$-expansion $S^{\prime}$ of $S^{o}$ such that

$$
\begin{gathered}
\mathrm{i} \mathrm{Eq} \vdash \mathrm{LJ} S \Leftrightarrow \mathrm{i} \mathrm{Eq}_{{ }^{\prime o}} S \Leftrightarrow \mathrm{iEq}_{k} \vdash_{i o} S \Leftrightarrow \\
\mathrm{iEq}_{k} \vdash_{i o} S^{k} \Leftrightarrow \mathrm{iEq}_{k} \vdash_{c o} S^{k} \Leftrightarrow \\
\mathrm{iEq}_{k} \vdash_{c o} S^{o} \Leftrightarrow \mathrm{iEq}_{k} \vdash_{i o} S^{o} \Leftrightarrow \mathrm{iEq}_{k} \vdash_{i o} S^{\prime} \Leftrightarrow \mathrm{iEq}_{k} \vdash_{c o} S^{\prime} .
\end{gathered}
$$

\subsection{The theory of groups}

For the theory of groups iGrp [?], which includes the equality axioms, the group axioms of $\mathrm{iGrp}^{k}$ become, when we leave out the standard parts ( $e$ for the unit and ${ }^{-1}$ for the inverse):

$$
\begin{aligned}
& \Rightarrow\langle k, x \cdot e\rangle=\langle k, e \cdot x\rangle=\langle k, x\rangle \\
& \Rightarrow\langle k, x \cdot(y \cdot z)\rangle=\langle k,(x \cdot y) \cdot z\rangle \\
& \Rightarrow\left\langle k, x \cdot x^{-1}\right\rangle=\left\langle k, x^{-1} \cdot x\right\rangle=\langle k, e\rangle .
\end{aligned}
$$

Observe that $\mathrm{iGrp}_{k}$ derives $\mathrm{iGrp}{ }^{k}$. Sometimes apartness is included in the theory of groups, in which case the theory is not atomic anymore. Due to lack of space we only treat the theory of groups without apartness here. In a subsequent paper we will show that similar results as the one below do hold for the theory of apartness, as well as for the theory of groups in which apartness is included. Since iGrp ${ }^{k}$ is universal, and the theory of groups is atomic, and $\mathrm{iGrp}_{k}$ derives iGrp $^{k}$, by Corollary 3 and Corollary 5 we can conclude

Corollary 8 For $S$ in $\mathcal{L}, k \in \mathcal{L}_{s}$ there exists a quantifier free $\wedge \vee$-expansion $S^{\prime}$ of $S^{o}$ such that

$$
\begin{aligned}
& \mathrm{iGrp} \vdash_{\mathrm{LJ}} S \Leftrightarrow \mathrm{iGrp} \vdash_{i o} S \Leftrightarrow \mathrm{iGrp}^{k} \vdash_{i o} S^{k} \Leftrightarrow \mathrm{iGrp}^{k} \vdash_{c o} S^{k} \Leftrightarrow \\
& \mathrm{iGrp}^{k} \vdash_{c o} S^{o} \Leftrightarrow \mathrm{iGrp}^{k} \vdash_{i o} S^{o} \Leftrightarrow \mathrm{iGrp}^{k} \vdash_{i o} S^{\prime} \Leftrightarrow \mathrm{iGrp}^{k} \vdash_{c o} S^{\prime} .
\end{aligned}
$$

For $S$ in $\mathcal{L}, k \in \mathcal{L}_{s}$ there exists a quantifier free $\wedge \vee$-expansion $S^{\prime}$ of $S^{o}$ such that

$$
\begin{gathered}
\mathrm{iGrp} \vdash_{\mathrm{LJ}} S \Leftrightarrow \mathrm{iGrp} \vdash_{i o} S \Leftrightarrow \mathrm{iGrp}_{k} \vdash_{i o} S \Leftrightarrow \\
\mathrm{iGrp}_{k} \vdash_{i o} S^{k} \Leftrightarrow \mathrm{iGrp}_{k} \vdash_{c o} S^{k} \Leftrightarrow \\
\mathrm{iGrp}_{k} \vdash_{c o} S^{o} \Leftrightarrow \mathrm{iGrp}_{k} \vdash_{i o} S^{o} \Leftrightarrow \mathrm{iGrp}_{k} \vdash_{i o} S^{\prime} \Leftrightarrow \mathrm{iGrp}_{k} \vdash_{c o} S^{\prime} .
\end{gathered}
$$




\subsection{Other theories}

The method of transforming Theorem 4, which holds for all theories, into results that are similar in spirit to Theorem 5 and Corollary 5 , can be applied to many other constructive theories and intermediate logics. However, since many of these theories fail to be atomic additional techniques have to be used to establish such results. For this reason these applications, especially to Heyting Arithmetic and the theory of apartness, will be treated seperately in a subsequent paper.

\section{Decidability}

The order calculi LJO and LKO can easily be transformed into equivalent calculi that have cut elimination. As a consequence we obtain the decidability of the quantifier free fragments of these logics. Thus also the derivability of Herbrand expansions of orderized formulas is decidable in these systems. The idea is simple: we replace the set of axioms in the calculi by their cut-hull, that is, by the closure of the axiom set under the cut rule. We are convinced that similar theorems also hold for the theory of equality and the theory of groups, but due to lack of space we have postponed proofs of these facts to a subsequent paper.

\section{$10 \mathrm{LJO}$ and LKO}

For a set of formulas $\Gamma$ we write $k \preccurlyeq^{\prime} l$ if $K k \in \Gamma$ or $k=\iota$, and $K l \in \Gamma$ or $l=\iota$, and $k=l$ or $k=\iota$ or $k \preccurlyeq l \in \Gamma$. $\preccurlyeq_{\Gamma}$ is the transitive closure of $\preccurlyeq_{\Gamma}^{\prime}$. Observe that $K k \in \Gamma$ if and only if $k \preccurlyeq_{\Gamma} k$, except when $k$ equals $\iota$, in which case $\iota \preccurlyeq_{\Gamma} \iota$ holds for all $\Gamma$. Note that $k \preccurlyeq_{\Gamma} l$ implies $k \preccurlyeq_{\Pi} l$ for every $\Pi$ that contains all formulas of the form $m \preccurlyeq n$ and $K m$ that belong to $\Gamma$. We write $\preccurlyeq \Gamma \Pi$ for $\preccurlyeq \Gamma \cup \Pi$ and $\preccurlyeq_{\Gamma A}$ for $\preccurlyeq \Gamma \cup\{A\}$.

From now on " $t(\bar{x})$ is a term in $\mathcal{L} \cup\left\{\epsilon, x_{1}, \ldots, x_{n}\right\}$ " means that $t(\bar{x})$ contains no other symbols than the ones in $\mathcal{L} \cup\left\{\epsilon, x_{1}, \ldots, x_{n}\right\}$. We say that $\Gamma$ supports $\langle k, x\rangle$ if $x=t(\bar{y})$ for some term $t(\bar{y})$ in $\mathcal{L} \cup\{\epsilon, \bar{y}\}$, and $\Gamma$ contains formulas $E\left\langle k_{i}, y_{i}\right\rangle$ such that $k_{i} \preccurlyeq_{\Gamma} k$. Observe that if $\Gamma$ supports $\langle k, x\rangle$, then so does $\Pi$ for every $\Pi$ that contains all formulas of the form $m \preccurlyeq n, K m$ and $E\langle l, y\rangle$ that belong to $\Gamma$.

Let $\mathrm{LJO}^{\prime}$ be LJO without weakening and contraction in which $\Gamma$ is added to the antecedent of the axioms $A x$ and $\mathrm{L} \perp$, and $\mathrm{L} \rightarrow$ and $\mathrm{L} \forall$ are replaced by

$$
\mathrm{L}^{\prime} \rightarrow \frac{\Gamma, A \rightarrow B \Rightarrow C \quad \Gamma, B \Rightarrow C}{\Gamma, A \rightarrow B \Rightarrow C} \quad \frac{\Gamma, \forall x A x, A t \Rightarrow C}{\Gamma, \forall x A x \Rightarrow C}
$$


and in which $A x_{o}$ is replaced by the following axioms

$$
\begin{aligned}
\text { (1) } \Gamma \Rightarrow K \iota \quad(2) \Gamma \Rightarrow k \preccurlyeq l \quad(\text { if } k \preccurlyeq \Gamma l) \\
\text { (3) } \Gamma, P\langle k, \bar{x}\rangle \Rightarrow P\langle l, \bar{x}\rangle \\
\quad\left(k \preccurlyeq \Gamma l, \Gamma \text { supports }\left\langle k, x_{i}\right\rangle, P \text { in } \mathcal{L}\right) \\
\text { (4) } \Gamma \Rightarrow E\langle l, t(\bar{x})\rangle
\end{aligned}
$$

$(t(\bar{x})$ is a term in $\mathcal{L} \cup\{\epsilon, \bar{x}\}, \Gamma$ supports $\langle l, t(\bar{x})\rangle)$

The part of LJO' without these axioms is the standard Gentzen calculus G3i for intuitionistic logic without weakening and contraction [?].

$\mathrm{LKO}^{\prime}$ is defined in a similar way: it is LKO without weakening and contraction in which $\Gamma$ is added to the antecedent of the axioms $A x$ and $\mathrm{L} \perp$, and $\mathrm{R} \exists$ and $\mathrm{L} \forall$ are replaced by

$$
\frac{\Gamma \Rightarrow \Delta, \exists x A x, A t}{\Gamma \Rightarrow \Delta, \exists x A x} \quad \frac{\Gamma, \forall x A x, A t \Rightarrow \Delta}{\Gamma, \forall x A x \Rightarrow \Delta}
$$

and in which $A x_{o}$ is replaced by the same axioms as in the case of $\mathrm{LJO}^{\prime}$, except that $\Delta$ is added to all the succedents of the sequents.

Recall that for $\bar{x}=x_{1}, \ldots, x_{n}, P\langle k, \bar{x}\rangle$ is short for $P\left(\left\langle k, x_{1}\right\rangle, \ldots,\left\langle k, x_{n}\right\rangle\right)$ for $n$-ary predicates $P$. Axioms (1) and (2) correspond to the partial order axioms $A x_{p o}$ of LJO; the sequents $\Gamma \Rightarrow \iota \preccurlyeq \iota$ and $\Gamma, K k \Rightarrow \iota \preccurlyeq k$ and $\Gamma, K k \Rightarrow k \preccurlyeq k$ all follow from (2). Axiom (3) corresponds to the upwards persistency axiom, except for the case that $P$ equals $E$, but this case has been included in axiom (4), which the reader can check for himself. Axiom (4) corresponds to the existence axioms of LJO: note that it implies $\Rightarrow E\langle\iota, \epsilon\rangle$ as well as $K k \Rightarrow E\langle k, t\rangle$ for any closed term $t$ in $\mathcal{L} \cup\{\epsilon\}$. Clearly, $\mathrm{LJO}^{\prime}$ and $\mathrm{LKO}^{\prime}$ are equivalent to LJO and LKO.

Lemma 16 (Cut elimination)

LJO' and LKO' have cut elimination.

Proof From the standard proof of the cut elimination theorem for the variant of LJ in which contraction and weakening are built-in into the axioms [?], it follows that every proof in LJO' can be reduced to a proof in which the only cuts are cuts on axioms. Therefore it suffices to show that the set of axioms of LJO' $^{\prime}$ is closed under cuts. That is, if $\Gamma \Rightarrow A$ and $\Gamma, A \Rightarrow C$ are instances of certain axioms of $\mathrm{LJO}^{\prime}$, then the conclusion $\Gamma \Rightarrow C$ of an application of the cut

$$
\frac{\Gamma \Rightarrow A \quad \Gamma, A \Rightarrow C}{\Gamma \Rightarrow C}
$$

is an instance of an axiom too.

It is not difficult to see that this holds when one of the axioms is $A x$ or $\mathrm{L} \perp$, the argument can be found in the proof of cut elimination for G3i in [?]. Thus we only consider the case that both axioms are instances of the order axioms 
(1)-(4) of LJO'. Recall that we write $\preccurlyeq_{\Gamma A}$ for $\preccurlyeq_{\Gamma \cup\{A\}}$. Observe that if the left hypothesis is an instance of (1), and thus $A=K \iota$, it suffices to show that $k \preccurlyeq_{\Gamma A} l$ implies $k \preccurlyeq_{\Gamma} l$, which follows immediately from the definition of $\preccurlyeq$. If the right hypothesis is an instance of (1), then so is the conclusion of the cut. Therefore suppose that both hypotheses are instances of (2)-(4). We distinguish by cases. We suppose $A$ is principal in both hypotheses, because if it is not, the conclusion of the cut is an instance of the same axiom of which the right hypothesis is an instance.

First suppose the left hypothesis is an instance of (2), i.e. $A$ is of the form $m \preccurlyeq n$. Note that it suffices to show that $k \preccurlyeq_{\Gamma A}^{\prime} l$ implies $k \preccurlyeq_{\Gamma} l$. So suppose $k \preccurlyeq_{\Gamma A}^{\prime} l$. If $k \neq m$ or $l \neq n$, then $k \preccurlyeq_{\Gamma}^{\prime} l$ follows immediately. Thus suppose $k=m$ and $l=n$. Because the left hypothesis is an instance of (2) we have $m \preccurlyeq \Gamma n$. That is, $k \preccurlyeq_{\Gamma} l$.

Second, suppose the left hypothesis is an instance of (3), i.e. $A$ is of the form $P(\langle k, \bar{x}\rangle)$, for $P$ in $\mathcal{L}$. Since we assumed that the cut formula is principle in both hypotheses, the right hypothesis is an instance of (3) or (4). In case both hypotheses are instances of (3) we have the following cut

$$
\frac{\Gamma, P(\langle l, \bar{x}\rangle) \Rightarrow P(\langle k, \bar{x}\rangle) \quad \Gamma, P(\langle k, \bar{x}\rangle), P(\langle l, \bar{x}\rangle) \Rightarrow P\langle m, \bar{x}\rangle}{\Gamma, P(\langle l, \bar{x}\rangle) \Rightarrow P\langle m, \bar{x}\rangle}
$$

where $l \preccurlyeq_{\Gamma} k$, and $\Gamma$ supports all $\left\langle l, x_{i}\right\rangle$ and $\left\langle m, x_{i}\right\rangle$. Since $A$ is also principal in the right hypotheses, $k \preccurlyeq_{\Gamma} m$ or $l \preccurlyeq_{\Gamma} m$ holds. Hence $l \preccurlyeq_{\Gamma} m$. Thus the conclusion of the cut is an instance of (3).

If the left hypothesis is an instance of (3) and the right hypothesis is an instance of (4), then $A$ is not principal in the right hypothesis, and so the conclusion of the cut is an instance of (4).

Finally, suppose the left hypothesis is an instance of (4), i.e. $A$ is of the form $E\langle k, t(\bar{x})\rangle$, where $t(\bar{x})$ is a term in $\mathcal{L} \cup\{\epsilon, \bar{x}\}$, and $\Gamma$ supports $\langle k, t(\bar{x})\rangle$. Since we assumed the cut formula to be principal in both hypotheses, the right hypothesis is an instance of (3) or (4). In the former case we have the following cut

$$
\frac{\Gamma, P(\langle l, \bar{y}\rangle) \Rightarrow E\langle k, t(\bar{x})\rangle \quad \Gamma, P(\langle l, \bar{y}\rangle), E\langle k, t(\bar{x})\rangle \Rightarrow P(\langle m, \bar{y}\rangle)}{\Gamma, P(\langle l, \bar{y}\rangle) \Rightarrow P(\langle m, \bar{y}\rangle)}
$$

for some $P$ in $\mathcal{L}$, where $\Gamma$ supports $\langle k, t(\bar{x})\rangle$, and $\Gamma \cup\{E\langle k, t(\bar{x})\rangle\}$ supports $\left\langle l, y_{i}\right\rangle$ and $l \preccurlyeq_{\Gamma} m$. The only non trivial case occurs when $E\langle k, t(\bar{x})\rangle$ plays a role in the fact that $\Gamma$ supports $\left\langle l, y_{i}\right\rangle$. Thus if $y_{i}=r(t(\bar{x}), \bar{z})$ for some $r(t(\bar{x}), \bar{z})$ in $\mathcal{L} \cup\{\epsilon, \bar{x}, \bar{z}\}$. Note that whence $k \preccurlyeq_{\Gamma} l$. Since also $\Gamma$ supports $\langle k, t(\bar{x})\rangle$, it follows that it supports $\left\langle l, y_{i}\right\rangle$. Therefore the conclusion of the cut is an instance of (3). In case both hypotheses are instances of (4) we have the following cut

$$
\frac{\Gamma \Rightarrow E\langle k, t(\bar{x})\rangle \quad \Gamma, E\langle k, t(\bar{x})\rangle \Rightarrow E\langle l, s(\bar{y})\rangle}{\Gamma \Rightarrow E\langle l, s(\bar{y})\rangle}
$$

where $t(\bar{x})$ and $s(\bar{y})$ are terms in $\mathcal{L} \cup\{\epsilon, \bar{x}\}$ and $\mathcal{L} \cup\{\epsilon, \bar{y}\}$ respectively, and $\Gamma$ supports $\langle k, t(\bar{x})\rangle$ and $\Gamma \cup\{E\langle k, t(\bar{x})\rangle\}$ supports $\langle l, s(\bar{y})\rangle$. The only non trivial 
case occurs when $E\langle k, t(\bar{x})\rangle$ plays a role in the fact that $\Gamma$ supports $\langle l, s(\bar{y})\rangle$. Thus $t(\bar{x})$ equals some $y_{i}$ and $k \preccurlyeq_{\Gamma} l$. Since $\Gamma$ supports $\langle k, t(\bar{x})\rangle$ this implies that it supports $\langle l, s(\bar{y})\rangle$. Hence the conclusion of the cut is an instance of (4). ○

Corollary 9 The quantifier free fragments of LJO' and LKO' are decidable. Thus so are the quantifier free fragments of LJO and LKO.

Proof That the quantifier free fragment of LKO' $^{\prime}$ is decidable follows from the following two facts. All propositional rules in the calculus are invertible, meaning that the hypotheses of the rules are derivable if the conclusion is. And the complexity of the principal formulas in the hypotheses of a rule is lower than that of the principal formula in the conclusion. Therefore bottom-up proof search for quantifier free formulas is a finite process, from which decidability follows.

The argument for $\mathrm{LJO}^{\prime}$ is similar, but one has to take into account that its left implication rule $\mathrm{L}^{\prime} \rightarrow$ is not invertible. There are many results in the literature that show how this problem can be dealt with for the system G3i; the number of applications of L' $\rightarrow$ to the same formula in a proof can be bounded in various ways, and from this the decidability of the quantifier free fragment of the logic follows [?]. These results and their proofs carry over to LJO', and thereby the decidability of the quantifier free fragment of $\mathrm{LJO}^{\prime}$ is established.

Corollary 10 The derivability of Herbrand expansions of orderized sequents is decidable in LJO', LJO, LKO' and LKO.

Corollary $11 \mathrm{LJO}$ and LJO' have the disjunction property and the term existence property.

\section{References}

[1] M. Baaz and R. Iemhoff. Interpolation in existence logics. In Proceedings of LPAR 2005 - Lecture Notes in Computer Science, volume 3835, pages 697-711, 2005.

[2] M. Baaz and R. Iemhoff. On the proof theory of the existence predicate. In We will show them! Essays in honour of Dov Gabbay, volume 1. King's College Publications, 2005.

[3] M. Baaz and R. Iemhoff. Gentzen calculi for the existence predicate. Studia Logica, 82(1):7-23, 2006.

[4] M. Baaz and R. Iemhoff. Skolemization in intuitionistic logic. Annals of Pure and Applied Logic, 142:269-295, 2006.

[5] M. Baaz and R. Iemhoff. Orderization, Skolemization and constructive theories. 2007. In preparation.

[6] S.R. Buss. On Herbrand's theorem. In Logic and Computational Complexity, volume 960 of Lecture Notes in Computer Science, pages 195-209. 1995.

[7] S.R. Buss. Handbook of Proof Theory. Elsevier, 1998.

[8] M. Fitting. Resolution for intuitionistic logic. In Z. Ras and M. Zemankovaet, editors, International Symposium on Methodologies for Intelligent Systems 1987, pages 400-407. North-Holland, 1987. 
[9] G. Kreitz, J. Otten, S. Schmitt, and B. Pientka. Matrix-based constructive theorem proving. In Intellectics and Computational Logic: Papers in Honor of Wolfgang Bibel. Kluwer, 2000.

[10] G.E. Mints. An analogue of Hebrand's theorem for the constructive predicate calculus. Sov. Math. Dokl., 3:1712-1715, 1962.

[11] G.E. Mints. Hebrand's theorem for the predicate calculus with equality and function symbols. Sov. Math.,Dokl., 7:911-914, 1966.

[12] G.E. Mints. The Skolem method in intuitionistic calculi. Proc. Steklov Inst. Math., 121:73-109, 1972.

[13] G.E. Mints. Resolution strategies for the intuitionistic predicate logic. In B. Mayoh et al., editor, Constraint Programming. Proceedings of the NATO Advanced Study Institute, volume 131 of Comput. Syst. Sci., pages 289-311. Springer, 1994.

[14] G.E. Mints. Axiomatization of a Skolem function in intuitionistic logic. In M. et al. Faller, editor, Formalizing the dynamics of information, volume 91 of CSLI Lect. Notes, pages 105-114. 2000.

[15] G.E. Mints. Thoralf Skolem and the epsilon substitution method for predicate logic. Nord. J. Philos. Log., 1(2):133-146, 2000.

[16] D.S. Scott. Identity and existence in intuitionistic logic. In Fourman et al., editor, Applications of sheaves, Proc. Res. Symp. Durham 1977, volume 753 of Lect. Notes Math., pages 660-696, 1979.

[17] N. Shankar. Proof search in the intuitionistic sequent calculus. In D. Kapur, editor, Automated deduction-CADE-11, volume 607 of Lect. Notes Comput. Science, pages 522-536, Berlin, 1992. Springer.

[18] A.S. Troelstra and D. van Dalen. Constructivism in Mathematics. North-Holland, Amsterdam, 1988.

[19] L.A. Wallen. Automated Proof Search in Non-Classical Logics. MIT Press, 1990. 